\title{
BROADBAND IMPEDANCES OF ACCELERATING STRUCTURES: PERTURBATION THEORY *
}

\author{
S. HEIFETS \\ Stanford Linear Accelerator Center, Stanford University, \\ Stanford, California 94309
}

(Received 16 June 1992; in final form 1993)

\begin{abstract}
A perturbation theory for broadband impedance calculations has been developed, allowing evaluation of impedances for an accelerating structure of a rather arbitrary shape. General formulas are given for the longitudinal and transverse impedances. The method is checked by calculating impedances and comparing results with those for structures previously studied. Several new results, including impedance of a taper, are presented.
\end{abstract}

KEY WORDS: Electromagnetic Field Calculations, Impedances

\section{INTRODUCTION}

The interaction of a beam with its environment in accelerators is usually described in terms of the coupling impedances. There is a vast literature dedicated to the impedance calculations and their properties; see for example Reference 1. Most quantitive results have been obtained using numeric codes. ${ }^{2}$ Analytical results in most cases are limited to derivation of integral equations which have to be solved numerically; Kirchhoff's equations ${ }^{3}$ are an example of such equations. Although integral equations for an impedance may be useful for studying its general properties, numeric solution of the integral equations is hardly justified, since numerical codes based on the direct solution of Maxwell's equations with appropriate boundary conditions already exist and have proved to be very successful.

However, the estimates based on the integral equations may be extended to give explicit analytic results for structures of rather general form, especially in the highfrequency limit. This approach - being complementary to numerical calculations may be very fruitful for modern accelerators, where bunches are short compared to the beampipe radius (or, in other words, the frequency content extends well above the beampipe cutoff frequency). At high frequencies, it is possible to formulate a perturbation theory based on Kirchhoff's equations, analogous to the Born series in scattering theory. Essentially, a perturbation theory of this kind was used in the

\footnotetext{
* Work supported by Department of Energy contract DE-AC03-76SF00515.
} 
time domain by Novokhatsky ${ }^{4}$ in 1988 for derivation of the impedances of a step in a beampipe, and of a pillbox cavity with attached pipes. The author ${ }^{5}$ used a perturbation theory in 1989 for more-general structures in the frequency domain. The present paper is a revised version of an unpublished talk given at a KEK (Tsukuba, Japan) workshop in September 1990. The perturbative method is described in a systematic way, and is applied to study more cases - particularly, to study the impedance of a taper. Axial symmetry is implied in most cases stated otherwise, although the method may also be applied to study impedances of structures without axial symmetry.

The most difficult problem is formulation of a criterion of applicability of the method. The rough estimate of the parameter of expansion can be obtained by comparing sequential terms of the perturbation series, as it is usually done for Born's series. It is natural to expect that for small loss parameters, the parameter of expansion is small. However, even a small parameter of expansion does not guarantee that Born's series are convergent (usually they are not). We choose, therefore, another approach, comparing results of the method with numerical and analytical results known previously, and judging applicability by this comparison.

This paper is organized in the following way. In the beginning, we review the basic definitions. The idea of the method then is demonstrated, using a simple example from electrostatics for which the answer is well known. This method then reproduces Bethe's result ${ }^{6}$ for a field distorted by a hole in a conductive plane. The method is extended to electrodynamics, using Kirchhoff's integral equations, and applied to get Kurennoy's results ${ }^{7}$ for the longitudinal and transverse impedances of a hole in a straight pipe. In the next section the general expressions for the longitudinal and transverse impedances are derived, Equations (65) and (72), in the lowest nontrivial iteration for a axially symmetric beampipe with arbitrary variation of the radius. These formulas are used for particular geometries: a shallow cavity, a pillbox cavity with attached tubes, an array of such cavities, an abrupt variation of the pipe radius, a collimator, and a taper. This is followed by examples for the transverse impedance. The results are summarized in the conclusion.

\section{BASIC DEFINITIONS}

In the ultrarelativistic case, particles interact only through the EM fields excited in accelerating structures. The interaction may be described in terms of the wakefields in the time domain, or in terms of their Fourier components in the frequency domain. The longitudinal wake function $W_{l}(s)$ is related to the energy loss $\Delta E$ of a particle with the charge $e$, following a point-like bunch with the charge $q$ at the distance $s>0$, by

$$
\Delta E=-e q W_{l}(s)=e \int d z E^{z}[z, r, t=(z+s) / v],
$$

where $E^{z}$ is the longitudinal component of the electric field of the leading bunch.The longitudinal impedance then is defined as the synchronous harmonic of $E_{z}$ : 


$$
\begin{aligned}
Z_{l}(k) & =-(1 / q) \int d z E_{\omega}^{z}(z, r) \exp \{-i k z / \beta\} \\
W_{l}(s) & =\int(d \omega / 2 \pi) Z_{l}(k) \exp \{-i k s / \beta\} .
\end{aligned}
$$

The loss factor is given by the convolution of the Fourier component of the bunch density with the wake $W_{l}(s)$. For a Gaussian bunch, the loss factor is

$$
k_{l}=2 \int_{-\infty}^{\infty} d k \exp \left\{-k^{2} \sigma^{2}\right\} \operatorname{Re}\left(Z_{l}(k) / Z_{0}\right)
$$

where $Z_{0}=4 \pi / c=377 \Omega$ and $\sigma$ is the rms bunch length.

The transverse wake potential $W_{\perp}(s)$ is related to the transverse momentum $\Delta p_{\perp}=$ $e q r_{0} W_{\perp}(s)$ experienced by the trailing particle due to the field excited by the leading bunch moving parallel to the $z$ axes with the offset $r_{0}$. The transverse impedance $Z_{\perp}(k)$ is proportional to the synchronous harmonic of the transverse force and is a frequency harmonic of the transverse wake $W_{\perp}(s)$ :

$$
\begin{aligned}
& Z_{\perp}(k)=-\left(i / q r_{0}\right) \int_{-\infty}^{\infty} d z \exp \{-i k z / \beta\}\left[\vec{E}_{\omega}+(\vec{v} / c) \times B_{\omega}\right]_{\perp} \\
& W_{\perp}(s)=i \int(d \omega / 2 \pi) Z_{\perp}(k) \exp \{-i k s / \beta\}
\end{aligned}
$$

According to the Panofsky-Wenzel theorem, $Z_{\perp}$ is related to the longitudinal impedance:

$$
k Z_{\perp}=\left(1 / r_{0}\right)\left(\partial Z_{l} / \partial r\right) .
$$

This follows from the Maxwell equation $\vec{\nabla} \times \vec{E}=i k \vec{H}$ and the definitions in Equations (2) and (4).

Note also the following analytic properties of the impedance:

$$
Z_{l}\left(-k^{*}\right)=Z_{l}^{*}(k), \quad Z_{\perp}\left(-k^{*}\right)=-Z_{\perp}^{*}(k) .
$$

Therefore, the problem of impedance calculation is reduced to the problem of defining EM fields excited by a bunch in an accelerating structure.

The current density of a particle in free space with a charge $e$ moving parallel to the $z$ axis, with offset $r_{0}$ in the direction of the $x$ axis, has Fourier components

$$
\vec{j}_{\omega}^{p}=\int d t \exp \{i \omega t\} \vec{j}^{p}=e \hat{z} \delta(\phi)\left[\delta\left(r-r_{0}\right) / r\right] \exp \{i k z / \beta\} .
$$


Here $\hat{z}$ is a unit vector along the $z$ axis, and the azimuthal angle $\phi=0$ on the $x$ axis. This current produces EM fields given in terms of the potential $\phi_{\omega}$ :

$$
\begin{gathered}
E_{z}=\left[k^{2}+\left(\partial^{2} / \partial z^{2}\right)\right] \phi_{\omega}, \quad E_{\phi}=(1 / r)\left(\partial^{2} \phi_{\omega} / \partial \phi \partial z\right), \quad E_{r}=\left(\partial^{2} \phi_{\omega} / \partial r \partial z\right), \\
H_{z}=0, \quad H_{\phi}=i k\left(\partial \phi_{\omega} / \partial r\right), \quad H_{r}=-(i k / r)\left(\partial \phi_{\omega} / \partial \phi\right),
\end{gathered}
$$

where $k=\omega / c$ and, for $r>r_{0}$, the potential is

$$
\begin{aligned}
\phi_{\omega}= & -(2 i e / k c) \exp \{i k z / \beta\} \sum \exp \{i m \phi\} \\
& \times\left\{\delta_{m 0} \ln (k r / 2 \gamma)-\left[\left(1-\delta_{m 0}\right) / 2|m|\right]\left(r_{0} / r\right)^{|m|}\right\} .
\end{aligned}
$$

The EM field of an ultrarelativistic particle moving inside a straight, circular, cylindrical beampipe with an ideally conductive wall is given by Equation (7), where

$$
\begin{aligned}
\phi_{\omega}= & (2 i e / k c) \exp \{i k z / \beta\} \sum \exp \{i m \phi\} \\
& \times\left\{\delta_{m 0} \ln (a / r)+\left[\left(1-\delta_{m 0}\right) / 2|m|\right]\left[\left(r_{0} / r\right)^{|m|}-\left(r r_{0} / a^{2}\right)^{|m|}\right]\right\} .
\end{aligned}
$$

The field is zero outside the pipe and in the walls.

The field in the pipe for the monopole mode $m=0$,

$$
E_{r}=(2 e / \beta c r) \exp \{i k z / \beta\}, \quad H_{\phi}=\beta E_{r},
$$

is, with accuracy $o\left(1 \gamma^{2}\right)$, the same as the field of a particle in free space. Therefore in the limit $\gamma \rightarrow \infty$ there is no interaction of a particle with the beampipe and the impedances are zero. The result for the longitudinal impedance follows also from the fact that the radial component of the Poynting vector in this case is zero. For the dipole mode $|m|=1$ the field in the pipe is

$$
\begin{aligned}
& E_{z}=H_{z}, \\
& E_{\phi}=-H_{r}=E_{1} \sin (\phi)\left[1-\left(r^{2} / a^{2}\right)\right], \\
& E_{r}=H_{\phi}=E_{1} \cos (\phi)\left[1+\left(r^{2} / a^{2}\right)\right],
\end{aligned}
$$

where

$$
E_{1}=\left(2 e r_{0} / c r^{2}\right) \exp \{i k z / \beta\},
$$

giving zero transverse impedance, because the electric and magnetic components of the Lorenz force cancel each other with $(1 / \gamma)^{2}$ accuracy. 
This is not the case when the beampipe geometry varies with $z$. In the following pages we discuss a method to calculate impedances for rather general variation of the beampipe cross section. For simplicity, however, we assume axial symmetry, but leave the beampipe radius $a(z)$ an arbitrary function of $z$.

\section{ILLUSTRATION: THE METHOD FOR THE ELECTROSTATIC FIELD}

The problem of calculating EM fields excited by a bunch in an accelerating structure of arbitrary shape is rather complicated. However, the fields are usually small compared to the field of the bunch. It is natural to look for a perturbation method that will allow calculation of the induced field by iteration.

Let us start with an example that illustrates this approach. Consider a well-known electrostatic problem: find the field of a point-like charge $e$ placed at distance $z=a$ from an ideal ${ }^{1}$ conducting $x, y$ plane. The field potential for $z>0$ is a superposition of the potential $\phi_{\text {ext }}$ of a charge in free space,

$$
\phi_{\mathrm{ext}}(r, z)=e /\left[(z-a)^{2}+r^{2}\right]^{1 / 2}, \quad r^{2}=x^{2}+y^{2},
$$

and the potential of the image charge $-e$ at $z=-a$ :

$$
\phi_{0}(r, z)=\left\{e /\left[(z-a)^{2}+r^{2}\right]^{1 / 2}\right\}-\left\{e /\left[(z+a)^{2}+r^{2}\right]^{1 / 2}\right\} .
$$

This result may be obtained using Green's theorem ${ }^{3}$, which defines the field within a volume in terms of the field on the surface encompassing the volume:

$$
\phi(\vec{R})=\phi_{\mathrm{ext}}(\vec{R})+\int\left(d \vec{S}^{\prime} / 4 \pi\right)\left[G\left(\vec{R}, \vec{R}^{\prime}\right) \vec{\nabla}^{\prime} \phi\left(\vec{R}^{\prime}\right)-\phi\left(\vec{R}^{\prime}\right) \nabla^{\prime} G\left(\vec{R}, \vec{R}^{\prime}\right)\right] .
$$

The first term here, $\phi_{\text {ext }}$, is the field of a charge within the volume if there is any. The Green's function of the Laplace equation is simply $G\left(\vec{R}, \vec{R}^{\prime}\right)=1 /\left|\left(\vec{R}-\vec{R}^{\prime}\right)\right|$. The surface element $d \vec{S}=\vec{n} d S$, where $\vec{n}$ is a unit normal vector pointed to the outside of the volume. The vector $\vec{R}=(z, \vec{r})$, where the 2-D tangential $\vec{r}$ is orthogonal to $\vec{n}$. Derivatives on the surface are understood as the limit value of the derivatives calculated at the inside the volume.

The boundary condition on the conductive wall is $\phi=0$. Choose the surface of integration on the metallic boundaries, and the last term in Equation (15) vanishes. The remaining integral may be interpreted as the field of the induced surface charge, with the surface density proportional to $\vec{n} \vec{\nabla} \phi$ taken on the surface $z=0$.

Solve Equation (15) by iterations: $\phi=\phi^{(0)}+\phi^{(1)}+\ldots$. In the zeros approximation, $\phi^{(0)}=\phi_{\text {ext }}$. In the $n$th approximation

$$
\phi^{(n)}(\vec{R})=\int\left(d \vec{S}^{\prime} / 4 \pi\right) G \vec{\nabla} \phi^{(n-1)}(\vec{r}, 0), \quad n=1,2, \ldots,
$$


starting with $\phi^{(0)}=\phi_{\text {ext }}$. The integral for $n=1$ can be calculated easily using the integral representation of the Green's function:

$$
G(\vec{R})=\left[4 \pi /(2 \pi)^{3}\right) \int\left(d \vec{q} / q^{2}\right) \exp \{i \vec{q} \vec{R}\}
$$

This gives

$$
\phi^{(1)}(\vec{R})=-(1 / 2) e /\left[(z+a)^{2}+r^{2}\right]^{1 / 2} .
$$

In the next iteration,

$$
\phi^{(2)}(\vec{R})=-(1 / 2) \phi^{(1)}
$$

and so forth.

The series converges:

$$
\phi(\vec{R})=\phi_{\mathrm{ext}}(\vec{R})-e /\left[(z+a)^{2}+r^{2}\right]^{1 / 2}\left[(1 / 2)+(1 / 2)^{2}+(1 / 2)^{3} \ldots\right],
$$

giving the correct answer, Equation (14).

Note that although the final result satisfies the boundary condition, the result of any finite number of iterations does not. Hence, the solution of the Laplace equation is exact for each iteration, but the boundary conditions are satisfied only approximately.

The same method applied to a magnetostatic problem displays similar properties: the series converges, giving the right answer. Here, also, boundary conditions are satisfied only approximately for any finite number of iterations.

Let us make the problem a little more complex by adding a small round hole with radius $b$ in the conducting plane. Both the center of the hole and the charge are at $x=y=0$. The field is described by the potential $\Phi$,

$$
\begin{array}{r}
\Phi=\phi_{0}+\phi, \quad \text { for } \quad z>0, \\
\Phi=\psi, \quad \text { for } \quad z<0,
\end{array}
$$

where $\phi_{0}$ is the solution of the problem without the hole given by Equation (14) for $z>0$, and $\phi_{0} \equiv 0$ for $z<0$. The functions $\phi, \psi$ are to be found by perturbations.

With the surface of integration in the plane $z=0$, Green's theorem takes the form

$$
\begin{gathered}
\phi(\vec{R})=-\int\left(d \vec{S}^{\prime} / 4 \pi\right)\left[G\left(\vec{R}, \vec{R}^{\prime}\right)\left(\partial \phi\left(\vec{R}^{\prime}\right) / \partial z^{\prime}\right)\right]_{z^{\prime}=0} \\
-(\partial / \partial z) \int_{h}\left(d \vec{S}^{\prime} / 4 \pi\right)\left[G\left(\vec{R}, \vec{R}^{\prime}\right) \phi\left(\vec{r}^{\prime}, z^{\prime}\right)\right]_{z^{\prime}=0} \\
\psi(\vec{R})=\int\left(d \vec{S}^{\prime} / 4 \pi\right) G\left(\vec{R}, \vec{R}^{\prime}\right)\left(\partial \psi\left(\vec{R}^{\prime}\right) / d z^{\prime}\right)+(\partial / \partial z) \int_{h}\left(d \vec{S}^{\prime} / 4 \pi\right) G\left(\vec{R}, \vec{R}^{\prime}\right) \psi .
\end{gathered}
$$


The last integrals in Equations (20) and (21) are over the opening of the hole where, generally, $\Phi \neq 0$. These integrals can be considered as describing perturbation. The equations are homogeneous and have the trivial solution $\phi=\psi=0$. However, this solution is not acceptable, because it does not satisfy continuity of the derivative $\partial \Phi / \partial z$ at the opening.

The conditions of continuity on the hole are

$$
\psi=\phi, \quad(\partial \psi / \partial z)=\left(\partial \phi_{0} / \partial z\right)+(\partial \phi / \partial z) .
$$

The solution of Equations (20) and (21) is given by series

$$
\phi=\sum_{1}^{\infty} \phi_{n}, \quad \psi=\sum_{1}^{\infty} \psi_{n}
$$

The series for $\phi$ and $\psi$ are bootstraped by the conditions of Equation (22) in such a way that $\psi_{n}$ are driven by the derivatives $\partial \phi_{n} / \partial z$ and $\phi_{n}$ are driven by $\psi_{n}$ at the hole. The first-order $(n=1)$ correction is

$$
\begin{gathered}
\psi_{1}(\vec{R})=\int_{h}\left(d \vec{S}^{\prime} / 4 \pi\right) G\left(\vec{R}, \vec{R}^{\prime}\right)\left[\partial \phi_{0}\left(\vec{R}^{\prime}\right) / \partial z^{\prime}\right] \\
\phi_{1}(\vec{R})=-(\partial / \partial z) \int_{h}\left(d \vec{S}^{\prime} / 4 \pi\right) \psi_{1}\left(z^{\prime}\right) G\left(\vec{R}, \vec{R}^{\prime}\right),
\end{gathered}
$$

and for $n \geq 2$,

$$
\begin{aligned}
\psi_{n}(\vec{R})= & \left.\int_{h}\left(d \vec{S}^{\prime} / 4 \pi\right)\left\{G\left(\vec{R}, \vec{R}^{\prime}\right)\left[\partial \phi_{n-1}\left(\vec{R}^{\prime}\right) / \partial z^{\prime}\right]+\psi_{n-1}(\partial / \partial z) G\left(\vec{R}, \vec{R}^{\prime}\right)\right]\right\} \\
& +\int_{M} G\left(\vec{R}, \vec{R}^{\prime}\right)\left[\partial \psi_{n-1}\left(\vec{R}^{\prime}\right) / \partial z^{\prime}\right] \\
\phi_{n}(\vec{R})= & -\int_{h}\left(d \vec{S}^{\prime} / 4 \pi\right)\left\{G\left(\vec{R}, \vec{R}^{\prime}\right)\left[\partial \phi_{n-1}\left(\vec{R}^{\prime}\right) / \partial z^{\prime}\right]-\psi_{n}\left(z^{\prime}\right)\left[\partial G\left(\vec{R}, \vec{R}^{\prime}\right) / \partial z^{\prime}\right]\right\} \\
& -\int_{M}\left(d \vec{S}^{\prime} / 4 \pi\right) G\left(\vec{R}, \vec{R}^{\prime}\right)\left[\partial \phi_{n-1}\left(\vec{R}^{\prime}\right) / \partial z^{\prime}\right] .
\end{aligned}
$$

Here the indexes $(h)$ and $(M)$ mean integration over the hole and the metallic surfaces, correspondingly.

In the first approximation, we consider Equation (24), with $\phi_{0}$ given by Equation (14). For a small hole, $b \ll a$,

$$
E_{0}(0)=\left.\left(\partial \phi_{0} / \partial z\right)\right|_{z=0}=\left(2 e / a^{2}\right)
$$


and

$$
\psi_{1}(r, z)=\left(e b / a^{2}\right) \int_{0}^{\infty}(d q / q) J_{1}(q b) J_{1}(q r) \exp \{-q|z|\} .
$$

Consider the first-order correction $\phi_{1}$ to the potential due to the hole for $z>0$ at large distances $R \gg b$. Expanding $G\left(\vec{R}, \vec{R}^{\prime}\right)$, we obtain

$$
\begin{aligned}
\phi_{1}(\vec{R})= & (\partial / \partial z)(e b / R)(\partial / \partial a) \\
& \times \int_{h}\left(d S^{\prime} / 4 \pi a\right)\left[1+\left(\vec{r} \vec{r}^{\prime} / R^{2}\right)+\ldots\right] \int_{0}^{\infty}(d q / q) J_{1}(q b) J_{0}\left(q r^{\prime}\right) .
\end{aligned}
$$

The term $\vec{r} \vec{r}^{\prime} / R^{2}$ does not contribute. The rest of the integral is easy to calculate. This gives the field of an electric dipole $d$ :

$$
\phi_{1}(\vec{R})=-\left(z d / R^{3}\right), \quad d=(2 / 3 \pi)\left(e b^{3} / a^{2}\right),
$$

which is exactly the Bethe ${ }^{6}$ result $d=E_{0} b^{3} / 3 \pi$, where the unperturbed field $E_{0}$ at the opening is given by Equation (26).

Note that the boundary condition for $z<0$ is not satisfied and has to be corrected in the next approximation.

\section{KIRCHHOFF'S EQUATION - IMPEDANCE OF A HOLE}

We use the same method of iteration to solve the exact integral equation of the electrodynamics. Kirchhoff's integral equations ${ }^{3}$ are the analog of the Green's theorem considered above:

$$
\begin{aligned}
\vec{E}(\vec{R})= & \vec{E}_{b}-\int d S^{\prime}\left[\left(\vec{n}^{\prime} \vec{E}\right)\left(\vec{\nabla}^{\prime} G_{k}\right)+i k G_{k}\left(\vec{n}^{\prime} \times \vec{H}\right)\right] \\
& +\int d S^{\prime}\left[\vec{n}^{\prime}\left(\vec{E} \vec{\nabla}^{\prime}\right) G_{k}-\vec{E}\left(\vec{n}^{\prime} \vec{\nabla}^{\prime}\right) G_{k}\right] \\
\vec{H}(\vec{R})= & \vec{H}_{b}+\int d S^{\prime}\left[\vec{n}^{\prime}\left(\vec{H} \vec{\nabla}^{\prime}\right) G_{k}-\vec{H}\left(\vec{n}^{\prime} \vec{\nabla}^{\prime}\right) G_{k}\right] \\
& -\int d S^{\prime}\left[\left(\vec{n}^{\prime} \vec{H}\right)\left(\vec{\nabla}^{\prime} G_{k}\right)+i k\left(\vec{n}^{\prime} \times \vec{E}\right) G_{k}\right] .
\end{aligned}
$$

Here $\vec{n}$ is a unit vector normal to the surface pointed outside of the volume, and $G_{k}$ is the Green's function of the wave equation

$$
\left(\Delta+k^{2}\right) G_{k}\left(\vec{R}, \vec{R}^{\prime}\right)=-\delta\left(\vec{R}-\vec{R}^{\prime}\right) ;
$$


$\vec{E}_{b}, \vec{H}_{b}$ are the fields excited by the beam, with the charge density $\rho$ and current density $\vec{j}$ within the volume in the consideration:

$$
\begin{aligned}
\vec{E}_{b} & =4 \pi \int d V^{\prime}\left[\left(\vec{\nabla}^{\prime} G_{k}\right) \rho+i(k / c) G_{k} \vec{j}\left(\vec{R}^{\prime}\right)\right], \\
\vec{H}_{b} & =(4 \pi / c) \int d V^{\prime} \vec{j} \times\left(\vec{\nabla}^{\prime} G_{k}\right) .
\end{aligned}
$$

The Green's function $G_{k}(R)=\exp \{i k R\} /(4 \pi R)$ has the integral representation

$$
G_{k}\left(\vec{R}, \vec{R}^{\prime}\right)=\int\left\{d \vec{q} /\left[(2 \pi)^{3}\right]\right\}\left(\exp \left\{i \vec{q}\left(\vec{R}-\vec{R}^{\prime}\right)\right\}\right) /\left(q^{2}+k^{2}\right),
$$

or, in the cylindrical coordinates $(r, \phi, z)^{18}$,

$$
G_{k}\left(\vec{R}, \vec{R}^{\prime}\right)=(i / 8 \pi) \sum_{m} \exp \left\{i m\left(\phi-\phi^{\prime}\right)\right\} \int d p \exp \left\{i p\left(z-z^{\prime}\right)\right\} G_{m, p}\left(r, r^{\prime}\right),
$$

where

$$
G_{m, p}\left(r, r^{\prime}\right)=J_{m}(\Omega r) H_{m}^{(1)}\left(\Omega r^{\prime}\right) \theta\left(r^{\prime}-r\right)+J_{m}\left(\Omega r^{\prime}\right) H_{m}^{(1)}(\Omega r) \theta\left(r-r^{\prime}\right)
$$

Here $J_{m}, H_{m}^{(1)}$ are the Bessel and the Hankel's functions of the first kind, $\theta(r)$ is the step function, and

$$
\Omega=\left(k^{2}-p^{2}\right)^{1 / 2}, \quad \Omega(-p)=\Omega(p), \quad \Omega\left(-k^{*}\right)=-\Omega^{*}(k) .
$$

Note that $G_{m, p}(k)=G_{-m,-p}^{*}\left(-k^{*}\right)$.

To illustrate the perturbation method based on Kirchhoff's equations, consider the impedance of a slot in a straight beampipe.

It is easy to see that the field that is given by Equation (10) inside the pipe and is zero outside the pipe satisfies Kirchhoff's equations giveby by Equation (25).

Consider now a straight pipe with a small slot in it. Similarly to the electrostatic case, we first calculate the field outside the pipe due to the opening. In the first approximation, Equations (29) and (10) give the field outside of the pipe, $r>a$,

$$
\begin{aligned}
& \vec{E}(\vec{R})=-(2 e / c a) \vec{\nabla}_{\perp} \int_{h} d S^{\prime}\left[G_{k}\left(\vec{R}, \vec{R}^{\prime}\right) \exp \left\{i k z^{\prime}\right\}\right] \\
& \vec{H}(\vec{R})=-(2 e / c a)(\hat{z} \times \vec{\nabla}) \int_{h} d S^{\prime} \exp \left\{i k z^{\prime}\right\} G_{k}\left(\vec{R}, \vec{R}^{\prime}\right)
\end{aligned}
$$


The integrals here are over the surface area of the opening. The perturbation of the field inside the beampipe is then given by the last integrals in Equation (29), which vanished in the straight pipe due to boundary conditions. Requiring the tangential component of the electric field and the normal component of the magnetic field at the opening to be continuous, we define them from Equations (36) and obtain, in particular, $E_{z}$ inside the pipe:

$$
\begin{aligned}
E_{z}(\vec{R})= & (2 e / c a) \int_{h} d S^{\prime}\left(\left[\partial G_{k}\left(\vec{R}, \vec{R}^{\prime}\right) /\left(\partial r^{\prime}\right)\right]\right. \\
& \left.\times \int_{h} d S^{\prime \prime}\left(\partial / \partial z^{\prime \prime}\right) G_{k}\left(\vec{R}^{\prime}, \vec{R}^{\prime \prime}\right) \exp \left\{i k z^{\prime \prime}\right\}\right)_{r^{\prime}=r^{\prime \prime}=a}
\end{aligned}
$$

The longitudinal impedance is defined by Equations (2) and (37):

$$
\begin{aligned}
Z_{l}(k)= & -(2 / c a) \int d z \exp \{-i k z\} \int_{h} d S^{\prime}\left(\left[\partial G_{k}\left(\vec{R}, \vec{R}^{\prime}\right) /\left(\partial r^{\prime}\right)\right]\right. \\
& \left.\times \int_{h} d S^{\prime \prime}\left(\partial / \partial z^{\prime \prime}\right) G_{k}\left(\vec{R}^{\prime}, \vec{R}^{\prime \prime}\right) \exp \left\{i k z^{\prime \prime}\right\}\right)_{r^{\prime}=r^{\prime \prime}=a} .
\end{aligned}
$$

The integral over $d z$ can be calculated using Equation (33):

$$
\int d z \exp \{-i k z\} G_{k}\left(\vec{R}, \vec{R}^{\prime}\right)=(i / 4) \exp \left\{-i k z^{\prime} / \beta\right\} \sum_{m} \exp \left\{i m\left(\phi-\phi^{\prime}\right)\right\} G_{m, k / \beta}
$$

For $r^{\prime}>r$,

$$
G_{m, k / \beta}\left(r, r^{\prime}\right)=(2 i / \pi) \delta_{m, 0}\left[\ln \left(k r^{\prime} / 2 \gamma\right)+C\right]+\left[\left(1-\delta_{m, 0}\right) /(i \pi|m|)\right]\left(r / r^{\prime}\right)^{|m|}
$$

where $\delta_{m, n}=1$ for $m=n$ and zero otherwise, and $C=0.5772 \ldots$

The longitudinal impedance is dominated by the contribution of the monopole $m=0$ mode. For a slot with length $L$ and width $w=a \Delta \phi$, Equation (38) gives

$$
\begin{aligned}
Z_{l}(k)= & {\left[1 /(2 \pi)^{2} c\right] \int_{-L / 2}^{L / 2} d z^{\prime} d \phi d \phi^{\prime} \exp \left\{-i k z^{\prime}\right\} } \\
& \times\left[\exp \left\{i k z+i k\left[\left(z-z^{\prime}\right)^{2}+\Delta^{2}\right]^{1 / 2}\right\} /\left\{\left[\left(z-z^{\prime}\right)^{2}+\Delta^{2}\right]^{1 / 2}\right\}\right]_{z=-L / 2}^{L / 2}
\end{aligned}
$$


where $\Delta^{2}=4 a^{2} \sin ^{2}\left[\left(\phi-\phi^{\prime}\right) / 2\right]$. The integral over $d z^{\prime}$ can be written in the form

$$
\begin{aligned}
& \int_{-L / 2}^{L / 2} d z^{\prime} \exp \left\{-i k z^{\prime}\right\} \\
& \quad \times\left[\exp \left\{i k z+i k\left[\left(z-z^{\prime}\right)^{2}+\Delta^{2}\right]^{1 / 2}\right\} /\left[\left(z-z^{\prime}\right)^{2}+\Delta^{2}\right]^{1 / 2}\right]_{z=-L / 2}^{L / 2} \\
& \quad=2 i \int_{0}^{L}\left[d x \sin (k x) /\left(x^{2}+\Delta^{2}\right)^{1 / 2}\right] \exp \left\{i k\left(x^{2}+\Delta^{2}\right)^{1 / 2}\right\}
\end{aligned}
$$

The integrand here is finite at $x \rightarrow 0$. Hence, for a narrow slot $w \ll L, \Delta$ can be omitted. This gives the imaginary part of the impedance:

$$
\operatorname{Im} Z_{l}(k)=\left\{w^{2} /\left[(2 \pi)^{2} a^{2} c\right]\right\} \int_{0}^{2 k L}(d x / x) \sin (x) .
$$

If the slot is short $(k L \ll 1)$, then

$$
\operatorname{Im} Z_{l}(k)=Z_{0}\left\{k L w^{2} /\left[(2 \pi)^{3} a^{2}\right]\right\}
$$

reproduces Kurennoy's result. ${ }^{7}$

The impedance increases with $L$ for short slots $(k L \ll 1)$, and goes to a constant for $k L \gg 1$ :

$$
\operatorname{Im} Z_{l}(k)=\left\{Z_{0} \pi w^{2} /\left[4(2 \pi)^{3} a^{2}\right]\right\} .
$$

This result may be confusing because zero impedance may be expected for the infinitely long slot. It should be remembered, however, that this result, Equation (43), implies that the slot is long but finite, so that the field outside the beampipe is zero at infinity.

Dependence of $Z_{l}(k)$ on the length of a slot is shown in Figure 1.

The real part of the impedance describes energy losses, and is very small. ${ }^{8}$ Calculation of the real part of the impedance requires the next iteration, because the energy loss is related to the radiation through the hole, which cannot be described by the field given in Equation (36). These calculations have to be corrected by the field of induced charges on the outer surface of the beampipe, which can be obtained in the next approximation.

Similarly, consider the longitudinal dipole impedance of a narrow $(w \ll a)$ slot in a perfectly conductive pipe. Omitting intermediate calculations, we obtain from Equation (5) the transverse impedance

$$
Z_{\perp}(k)=\left\{i w^{2} /\left[(\pi)^{2} k c a^{4}\right]\right\} \int_{0}^{k L}(d x / x) \sin (x) \exp \{i x\}
$$




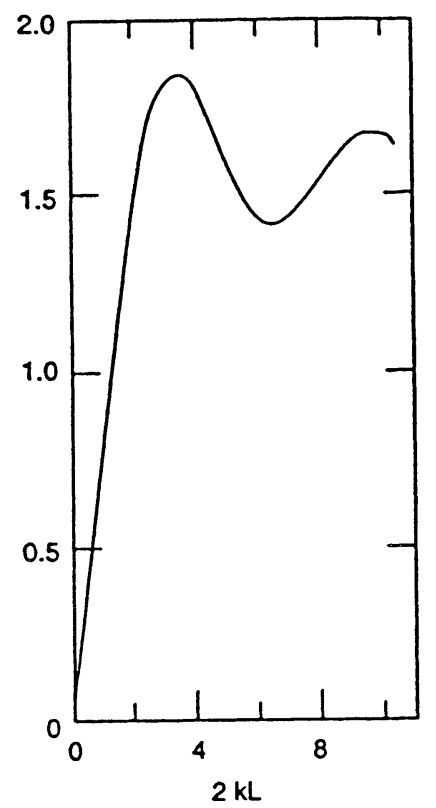

FIGURE 1: Dependence of the longitudinal impedance of a slot on the slot length: the integral in Equation (41) as a function of $2 k L$.

If $k L \ll 1$, the impedance does not depend on frequency: ${ }^{7}$

$$
Z_{\perp}(k)=i\left\{2 Z_{0} w^{2} L /\left[(2 \pi)^{3} a^{4}\right]\right\}
$$

If the bunch is long $(\sigma>L)$, the impedance for all frequencies within the bunch spectrum is described by this formula. The wake potential given by Equation (4) is proportional in this case to $\delta(s)$, and describes a transverse kick. The wake function depends on $s$ in the same way as the bunch charge density.

\section{LONGITUDINAL IMPEDANCE FOR A BEAMPIPE WITH ARBITRARY RADIUS VARIATION}

If a volume is bounded by metallic walls, it is useful to perform the integration at these walls where the normal component of the magnetic field and tangential components of the electric field are zero. In what follows we consider impedances due to variation 
in the beampipe radius with $z$. In this case, the last integrals in Equations (29) vanish due to the boundary conditions on the metallic walls. Kirchhoff's equations take the form

$$
\begin{aligned}
& \vec{E}(\vec{R})=\vec{E}_{b}-\int d S^{\prime}\left[\left(\vec{n}^{\prime} \vec{E}\right)\left(\vec{\nabla}^{\prime} G_{k}\right)+i k G_{k}\left(\vec{n}^{\prime} \times \vec{H}\right)\right], \\
& \vec{H}(\vec{R})=\vec{H}_{b}+\vec{\nabla} \times \int d S^{\prime}\left(\vec{H} \times \vec{n}^{\prime}\right) G_{k} .
\end{aligned}
$$

Equation (45) can be solved by iteration. It is worth noting again that, as in the example from electrostatics, we iterate Equations (45) where the exact boundary conditions are already imposed. If the equations were solved exactly, they would give the fields, satisfying the boundary conditions. However, the solution obtained with a finite number of iterations only approximately satisfies the boundary conditions.

Equations (45) can be written in terms of surface charge density $\sigma$ and surface current density $\vec{I}$ :

$$
\begin{aligned}
& \vec{E}=\vec{E}_{b}+4 \pi \int d S^{\prime}\left[\sigma\left(z^{\prime}\right)\left(\vec{\nabla}^{\prime} G_{k}\right)_{r^{\prime}=a\left(z^{\prime}\right)}+i(k / c) G_{k} \vec{I}\right], \\
& \vec{H}=\vec{H}_{b}+(4 \pi / c) \vec{\nabla} \times \int d S^{\prime} \vec{I} G,
\end{aligned}
$$

where $\sigma, \vec{I}$ are given by the fields at the boundary:

$$
4 \pi \sigma=-(\vec{E} \vec{n}), \quad(4 \pi / c) \vec{I}=-(\vec{n} \times \vec{H}) .
$$

The induced charge and current density $\rho, \vec{j}$ are related to $\sigma, \vec{I}: \rho=\sigma \delta(\eta), \vec{j}=\vec{I} \delta(\eta)$ where $\eta$ is the distance from the surface along the normal vector $\vec{n}(z)$,

$$
\eta=[r-a(z)] \cos \alpha, \quad \tan \alpha=a^{\prime}(z)=[d a(z) / d z] .
$$

Note that $\vec{n} \vec{I}=0$.

The continuity equation $i k c \rho=\vec{\nabla} \vec{j}$, which follows from Maxwell's equations, relates $\sigma$ and $\vec{I}$, giving

$$
I_{r}=a^{\prime}(z) I_{z}
$$

and

$$
i k c \sigma=[(\cos \alpha) / a](\partial / \partial z)\left(a I_{z} / \cos \alpha\right)+(1 / a)(\partial / \partial \phi) I_{\phi} .
$$

Equations (46) and (47) can be solved by iteration.

We give explicit expression for two azimuthal modes of the longitudinal impedance:

1. the monopole mode $m=0$, which dominates the longitudinal impedance, and 
2. the dipole mode $m=1$, which is related to the transverse impedance by the Panofsky-Wenzel theorem.

Only the induced part of the field $E_{z}$ is relevant for the impedance calculations. For a circular cylindrical beampipe with the radius $r=a(z)$, Equation (46) gives

$$
\begin{aligned}
E_{z}-E_{z}^{b}= & Z_{0} \int\left[d S^{\prime} / a\left(z^{\prime}\right)\right] \\
& \times\left\{( i / k ) \left[\cos \alpha\left(z^{\prime}\right)\left(\partial / \partial z^{\prime}\right)\left(a I_{z} / \cos \alpha\right)\right.\right. \\
& \left.\left.+\left(\partial I_{\phi^{\prime}} / \partial \phi^{\prime}\right)\right]\left(\partial G_{k} / \partial z\right)+i k a\left(z^{\prime}\right) G_{k} I_{z}\right\}
\end{aligned}
$$

Here

$$
d S^{\prime}=\left[a\left(z^{\prime}\right) d z^{\prime} d \phi^{\prime}\right] / \cos \alpha
$$

Using the definition in Equation (2) with Equation (45) for $E_{z}$, we obtain

$$
\begin{aligned}
Z_{l}(k)= & -\left(i Z_{0} / e k\right) \int\left[d S^{\prime} / a\left(z^{\prime}\right)\right] \\
& \times\left[\cos \alpha\left(z^{\prime}\right)\left(\partial / \partial z^{\prime}\right)\left(a I_{z} / \cos \alpha\right)+\left(\partial I_{\phi^{\prime}} / \partial \phi^{\prime}\right)\right]\left[\exp \{-i k z\} G_{k}\right]_{z=-\infty}^{\infty} \\
& -\left(Z_{0} / e\right) \int\left[d S^{\prime} / a\left(z^{\prime}\right)\right] \int d z \exp \{-i k z\} G_{k}\left\{i k a\left(z^{\prime}\right) I_{z}\left(z^{\prime}\right)-(1 / \beta)\right. \\
& \left.\times\left[\cos \alpha\left(z^{\prime}\right)\left(\partial / \partial z^{\prime}\right)\left(a I_{z} / \cos \alpha\right)+\left(\partial I_{\phi^{\prime}} / \partial \phi^{\prime}\right)\right]\right\}
\end{aligned}
$$

The first term vanishes unless $z^{\prime} \simeq z \rightarrow \infty$. At infinity the current is a surface current in a straight pipe

$$
\vec{I}=-\hat{z}(e / 2 \pi a) \exp \{i k z / \beta\}, \quad|z| \rightarrow \infty .
$$

Hence, $\partial a I_{z} / \partial z=0$ and the first term can be omitted.

The integration over $d z$ can be performed using Equations (36) and (39); then integration by parts over $d z^{\prime}$ gives, for the $m=0$ and $m=1$ modes,

$$
\begin{aligned}
Z_{l}^{(0)}(k)= & \left(Z_{0} / 2 \pi\right) \ln \left(a_{\infty} / a_{-\infty}\right) \\
& -\left(2 i k / e c \gamma^{2}\right) \int d S^{\prime}\left[\ln \left(k a\left(z^{\prime}\right) / 2 \gamma\right)+C\right] I_{z}\left(z^{\prime}\right) \exp \left\{-i k z^{\prime} / \beta\right\} \\
& +(2 / e c) \int d S^{\prime}\left[a^{\prime}\left(z^{\prime}\right) / a\left(z^{\prime}\right)\right] I_{z}\left(z^{\prime}\right) \exp \left\{-i k z^{\prime} / \beta\right\}
\end{aligned}
$$




$$
\begin{aligned}
Z_{l}^{(1)}(k)= & -\left(Z_{0} r r_{0} / 2 \pi\right)\left[\left(1 / a_{\infty}^{2}\right)-\left(1 / a_{-\infty}^{2}\right)\right] \cos \phi \\
& +\left[(2 i k r) /\left(e c \gamma^{2}\right)\right] \int\left[d S^{\prime} / a\left(z^{\prime}\right)\right] \exp \left\{-i k z^{\prime} / \beta\right\} \cos \left(\phi-\phi^{\prime}\right) I_{z} \\
& +(2 r / e c) \int\left[d S^{\prime} / a^{2}\left(z^{\prime}\right)\right] \exp \left\{-i k z^{\prime} / \beta\right\} \cos \left(\phi-\phi^{\prime}\right)\left[a^{\prime} I_{z}+\left(\partial I_{\phi^{\prime}} / \partial \phi^{\prime}\right)\right]
\end{aligned}
$$

\subsection{Zero approximation}

In the zero approximation, the surface current is defined by Equation (47) with the field Equations (10) and (11) of a bunch. For calculation of the longitudinal impedance, the offset $r_{0}$ can be set equal to zero. Then $I_{\phi}^{(0)}=0$, the two other components of $I^{(0)}$ are independent of azimuth, and

$$
Z_{0} \vec{I}_{z}^{(0)}=-(2 e / c a(z)) \exp \{i k z / \beta\} \cos \alpha .
$$

The impedance given by Equation (51) in the zeros approximation is

$$
\begin{aligned}
Z_{l}^{(0)}(k)= & \left(Z_{0} / 2 \pi\right) \ln \left(a_{\infty} / a_{-\infty}\right) \\
& -(1 / \pi c) \int d S\left[a^{\prime}(z) / a^{2}(z)\right] \cos \alpha(z) \\
& -\left(2 i k / e c \gamma^{2}\right) \int d S^{\prime} \exp \left\{-i k z^{\prime}\right\}[\ln (k a(z) / 2 \gamma)+C]
\end{aligned}
$$

The last term in Equation (54) is interesting only because it gives the impedance of the straight pipe per unit length:

$$
(d / d z) Z_{l}^{(0)}(k)=-\left[i k Z_{0} /\left(2 \pi \gamma^{2}\right)\right][\ln (k a / 2 \gamma)+C] .
$$

Usually, for $\gamma \gg 1$, it can be omitted.

The first two terms in Equation (54) give the zero-order result for the impedance of an abrupt change of a radius, usually called a step. ${ }^{12}$

For the "step-out," i.e., the case of a particle entering a wider pipe, the impedance is

$$
Z_{l}^{(0)}(k)=\left(Z_{0} / \pi\right) \ln [a(\infty) / a(-\infty)]
$$

It describes the change of the energy stored in the synchronous component of the field of a bunch due to difference of the beampipe radii at $z= \pm \infty$. For a "step-in," i.e., for a particle entering a narrower pipe, the two terms in Equation (52) cancel, giving zero impedance. This is in good agreement with the numerical calculations of Equation (13), which show that there is a small energy gain in this case. 
Note that Equation (56) is actually valid for an arbitrary dependence $a(z)$, including an abrupt change of the radius.

Similarly, the dipole mode of the longitudinal impedance given by Equation (52) in the zeroth approximation is given by the dipole component of the current $I^{(0)}$

$$
Z_{0} \vec{I}_{z}^{(0)}=-\left[4 e / c a^{2}(z)\right] \exp \{i k z / \beta\} \cos \phi \cos \alpha, \quad I^{(0)}=0 .
$$

The second term in Equation (52), according to Equation (5), gives the transverse impedance per unit length:

$$
(d / d z) Z_{\perp}=-\left(i Z_{0} / 2 \pi \gamma^{2} a^{2}\right) .
$$

The first and the last terms give for a step-in and a step-out, respectively:

$$
Z_{\perp}=-\left(Z_{0} / 2 \pi k\right)\left[\left(1 / a_{\infty}^{2}\right)-\left(1 / a_{-\infty}^{2}\right)\right][1 \mp(1 / 2)] .
$$

This impedance vanishes if the beampipe has equal radii at infinity. Also, it does not contribute to the wake field of a symmetric (Gaussian) bunch.

\subsection{First approximation}

The nontrivial part of the longitudinal impedance is given by the last term in Equation (51):

$$
Z_{l}^{0}(k)=\left(Z_{0} / 2 \pi e\right) \int d S\left[a^{\prime}(z) / a(z)\right] I_{z}^{(1)}(z) \exp \{-i k z / \beta\} .
$$

The current $I_{z}^{(1)}$ of the first approximation is defined by Equation (47):

$$
Z_{0} I_{z}^{(1)}=-H_{\phi}^{(1)} \cos \alpha
$$

where

$$
\vec{H}^{(1)}=\vec{\nabla} \times \vec{h}, \quad \vec{h}=Z_{0} \int d S^{\prime} \vec{I}^{(0)}\left(z^{\prime}, \phi^{\prime}\right) G_{k}\left(\vec{R}, \vec{R}^{\prime}\right) .
$$

Substituting the zero-order current

$$
Z_{0} \vec{I}^{(0)}=-[2 e / c a(z)] \exp \{i k z / \beta\}\left[\hat{r} a^{\prime}(z)+\hat{z}\right] \cos \alpha,
$$

we have $\vec{h}=-\left(\hat{r} h_{1}+\hat{z} h_{0}\right)$, where

$$
\begin{aligned}
& h_{0}=(2 e / c) \int\left[d S^{\prime} / a\left(z^{\prime}\right)\right] G_{k} \exp \left\{i k z^{\prime}\right\} \cos \alpha\left(z^{\prime}\right) \\
& h_{1}=(2 e / c) \int d S^{\prime}\left[a^{\prime}\left(z^{\prime}\right) / a\left(z^{\prime}\right)\right] G_{k} \exp \left\{i k z^{\prime}\right\} \cos \alpha\left(z^{\prime}\right) \cos \left(\phi-\phi^{\prime}\right) .
\end{aligned}
$$


It is convenient to write the component

$$
Z_{0} I_{z}^{(1)}=\left[\left(\partial h_{1} / \partial z\right)-\left(\partial h_{0} / \partial r\right)\right] \cos \alpha
$$

using a Green's function, as in Equation (33). The second term $\left(\partial h_{0} / \partial r\right)$ can be transformed, integrating $h_{0}$ by parts over dz'. After some algebra, we obtain

$$
\begin{aligned}
Z_{l}^{0}(k)= & \left(Z_{0} k / 8 \pi\right) \int d p \int d z a^{\prime}(z) \int d z^{\prime} a^{\prime}\left(z^{\prime}\right) \exp \left\{i(p-k)\left(z-z^{\prime}\right)\right\} \\
& \times\left\{\theta\left[a\left(z^{\prime}\right)-a(z)\right] J_{1}[\Omega a(z)] H_{1}^{(1)}\left[\Omega a\left(z^{\prime}\right)\right]+\theta[a(z)\right. \\
& \left.\left.-a\left(z^{\prime}\right)\right] J_{1}\left[\Omega a\left(z^{\prime}\right)\right] H_{1}^{(1)}[\Omega a(z)]\right\} .
\end{aligned}
$$

Equation (64) also can be written in the form

$$
\begin{aligned}
Z_{l}^{0}(k)= & -\left(i k Z_{0} / 2 \pi\right) \int d z a^{\prime}(z) \int d z^{\prime} a^{\prime}\left(z^{\prime}\right) \int d \phi^{\prime} \\
& \times\left[G_{k}\left(\vec{R}, \vec{R}^{\prime}\right)\right]_{r=a(z), r^{\prime}=a\left(z^{\prime}\right)} \cos \left(\phi-\phi^{\prime}\right) \exp \left\{-i k\left(z-z^{\prime}\right)\right\} .
\end{aligned}
$$

This expression is used below to study particular geometries $a(z)$.

The nontrivial part of the dipole longitudinal impedance is given by the last two terms in Equation (52):

$$
Z_{l}^{(1)}\left(k=(2 r / e c) \int\left[d S^{\prime} / a^{2}\left(z^{\prime}\right)\right] \exp \left\{-i k z^{\prime} / \beta\right\} \cos \left(\phi-\phi^{\prime}\right)\left[a^{\prime} I_{z}^{(1)}+\left(\partial I_{\phi^{\prime}}^{(1)} / \partial \phi^{\prime}\right)\right]\right.
$$

with the surface current determined in the first approximation,

$$
\vec{I}^{(1)}=-\left(1 / Z_{0}\right) \vec{n} \times \vec{H}^{(1)} .
$$

Here $\vec{H}^{(1)}$ is given by Equation (61) with the zero-order current of a beam with offset $r_{0}$,

$$
Z_{0} \vec{I}^{(0)}(z)=-[(\hat{r} \cos \phi+\hat{\phi} \sin \phi) \sin \alpha(z)+\hat{z} \cos \phi \cos \alpha(z)]\left(2 e r_{0} / c r^{2}\right) \exp \{i k z / \beta\} .
$$

After some calculations we have

$$
\begin{aligned}
\vec{h}= & -\left(i e r_{0} / 2 c\right) \int d p \int\left[d z^{\prime} / a\left(z^{\prime}\right)\right] \exp \left\{i k z^{\prime}+i p\left(z-z^{\prime}\right)\right\} \\
& \times\left\{\hat{z} G_{1 p} \cos \phi+a^{\prime}\left(z^{\prime}\right) G_{2 p}[\hat{r} \cos \phi+\hat{\phi} \sin \phi]\right\}
\end{aligned}
$$


Equations (66)-(69) give

$$
\begin{aligned}
Z_{l}^{(1)}(k)= & \left(i r r_{0} \cos \phi / 4 c\right) \int d p \int[d z / a(z)] \int\left[d z^{\prime} / a\left(z^{\prime}\right)\right] \\
& \times \exp \left\{i(k-p)\left(z^{\prime}-z\right)\right\}\left\{-a^{\prime}\left(z^{\prime}\right)\left[(1 / r)(\partial / \partial r) r G_{2 p}+(1 / r) G_{2 p}\right]\right. \\
& \left.+a^{\prime}(z)\left[\left(G_{1 p} / r\right)-\left(\partial G_{1 p} / \partial r\right)\right]+2 i p a^{\prime}(z) a^{\prime}\left(z^{\prime}\right) G_{2 p}\right\}_{r=a(z)} .
\end{aligned}
$$

This expression can be simplified by integration by parts. The final result for a pipe with equal radii at infinity is

$$
\begin{aligned}
Z_{l}^{(1)}(k)= & {\left[\left(k r r_{0} \cos \phi\right) / 2 c\right] \int d p \int[d z / a(z)]\left[d z^{\prime} / a\left(z^{\prime}\right)\right] } \\
& \times a^{\prime}(z) a^{\prime}\left(z^{\prime}\right) G_{2 p}\left(a(z), a\left(z^{\prime}\right)\right) \exp \left\{i(k-p)\left(z^{\prime}-z\right)\right\}
\end{aligned}
$$

This result can be rewritten as

$$
\begin{aligned}
Z_{l}^{(1)}(k)= & -Z_{0}\left[\left(i k r r_{0} \cos \phi\right) / 2 \pi\right] \int d \phi \cos 2\left(\phi-\phi^{\prime}\right) \int d z d z^{\prime} \\
& \times \exp \left\{-i k\left(z-z^{\prime}\right)\right\}\left\{\left[a^{\prime}(z) a^{\prime}\left(z^{\prime}\right)\right] /\left[a(z) a\left(z^{\prime}\right)\right]\right\} \\
& \times\left[G_{k}\left(\vec{R}, \vec{R}^{\prime}\right)\right]_{r=a(z), r^{\prime}=a\left(z^{\prime}\right)} .
\end{aligned}
$$

Transverse impedance is then given by Equation (5).

Equations (65) and (72) give a close form of the longitudinal and transverse impedances for a axially symmetric beampipe, with an arbitrary variation of the pipe radius $a(z)$. From these equations it is also easy to obtain the longitudinal and transverse wake fields.

In what follows, we apply these formulas to particular geometries.

\section{EXAMPLES OF LONGITUDINAL IMPEDANCE}

\subsection{Impedance of a shallow cavity and a small collimator}

We start with the simple case of a cavity with attached tubes:

$$
r(z)=a,|z|>g / 2, \quad r(z)=b,|z|<g / 2, b>a .
$$

Let us consider a shallow cavity $(b-a) \ll a, g \ll a, k\left[g^{2}+(b-a)^{2}\right]^{1 / 2} \ll 1$. The longitudinal impedance in this case is inductive. K.Bane ${ }^{9}$ approximates the results 
of simulations with the code TBCI for long bunches by the formula $Z_{l}^{(0)}=-i k L$, where the inductance

$$
L=\left(Z_{0} / 2 \pi\right)[g(b-a) / a] .
$$

Neglecting exponents in Equation (65), we obtain for this case

$$
\begin{aligned}
Z_{l}^{0}(k)= & -\left[i k Z_{0} /\left(2(2 \pi)^{2}\right)\right] \int_{a}^{b} d r \int_{a}^{b} d r^{\prime} \\
& \times \int d \phi \cos \phi\left\{\left[1 /\left(R^{2}\right)^{1 / 2}\right]-\left[1 /\left(g^{2}+R^{2}\right)^{1 / 2}\right]\right\}
\end{aligned}
$$

where $R^{2}=\left(r-r^{\prime}\right)^{2}+4 r r^{\prime} \sin ^{2}[\phi / 2]$. For a shallow cavity, a significant contribution is given by small angles $\phi \simeq(b-a) / a \ll 1, \phi \simeq g / a \ll 1$. The integrals can be evaluated, giving the inductance

$$
L=\left\{Z_{0}(b-a)^{2} /\left[(2 \pi)^{2} a\right]\right\} f(\lambda),
$$

where $\lambda=g /(b-a)$. For small $\lambda \ll 1$,

$$
f(\lambda)=4 \lambda \arctan (1 / \lambda)+\lambda^{2}(2 \ln \lambda+1),
$$

exactly giving K.Bane's result as in Equation (74); see also the discussion after Equation (83).

Consider now a shallow collimator

$$
r(z)=b,|z|>g / 2, \quad r(z)=a,|z|<g / 2, b>a,
$$

where $(b-a) \ll a, g \ll a, k\left[g^{2}+(b-a)^{2}\right]^{1 / 2} \ll 1$. Consideration of a shallow collimator is similar to that for a shallow cavity, with one significant difference. In deriving Equation (65), we implied that the field inside the conductor is zero. The current $I^{(1)}$ in Equation (60) is the current induced at the surfaces $z= \pm g / 2$ by the field generated by the zero-order currents, Equation (53). Clearly, for a collimator, $I^{(1)}$ at $z=-g / 2$ cannot be generated by the zero-order current on the opposite surface $z=+g / 2$, because the field inside the collimator is zero. The crosstalk between surfaces $z= \pm g / 2$ is generated only by the fields at the opening, and can be taken into account in the next approximations, as in the calculation of the impedance of a hole in a straight beampipe. Hence the impedance of a collimator is

$$
Z_{l}^{0}(k)=-\left[2 i k Z_{0} /(2 \pi)\right] \int d r \int d r^{\prime} \int d \phi \cos \phi G_{k}\left(r, r^{\prime}, z=z^{\prime}, \phi-\phi^{\prime}\right)
$$




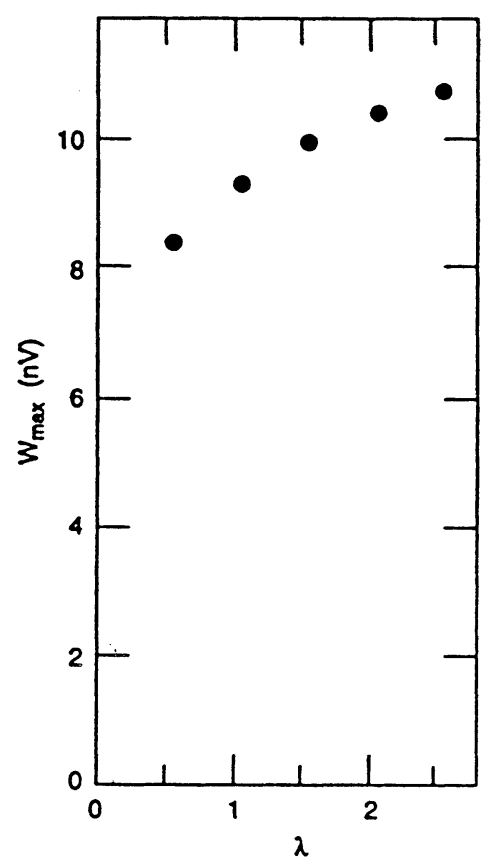

FIGURE 2: The TBCI results for the wake potential of a shallow collimator. Parameters: $\sigma=1 \mathrm{~cm}, b=4 \mathrm{~cm}$, $b-a=0.2 \mathrm{~cm}$. Perturbation theory gives the impedance constant in $g$.

Calculations for a shallow collimator can be performed explicitly, giving $Z_{l}^{(0)}=-i k L$ with the inductance

$$
L=\left[Z_{0}(b-a)^{2} / 4 \pi a\right]\{\ln [2 \pi a /(b-a)]+(3 / 2)\} .
$$

K. Bane ${ }^{8}$ describes TBCI results for this case by the formula

$$
L=\left[Z_{0}(b-a)^{2} /(\pi a)\right]
$$

which differs from Equation (80) by a factor of

$$
(1 / 4 \pi)\{\ln [2 \pi a /(b-a)]+(3 / 2)\} .
$$

Figure 2 gives TBCI results for $W_{\max }$ for an iris with an opening radius of $3.8 \mathrm{~cm}$, in a beampipe with a radius of $4.0 \mathrm{~cm}$. The iris thickness $g$ was changed in the range from 0.1 to $0.5 \mathrm{~cm}$. The bunch length is $1 \mathrm{~cm}$. The variation of $W_{\max }$ with $g$ is small.

It should be noted that simulations with TBCI of a shallow cavity and a small collimator are not simple, because they require a rather large number of mesh points. 


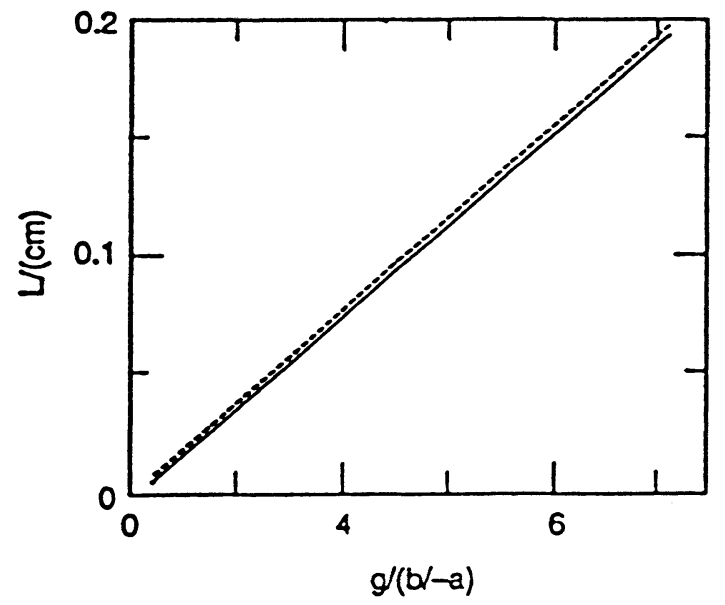

FIGURE 3: The inductance of a shallow cavity as a function of $\lambda=(b-a) / a$. The dashed line is calculated with Equation (74), based on TBCI results. Parameters of the cavity are $a=4 \mathrm{~cm}, b-a=0.2$.

\subsection{Impedance of a pillbox cavity with pipes and a step}

Consider a cavity in the high-frequency limit $k g \gg 1, k a \gg 1$. This case has been studied before ${ }^{10}$ and the answer is known. The real part of the impedance is

$$
\operatorname{Re} Z_{l}=\left(Z_{0} / 2 \pi a\right)(g / \pi k)^{1 / 2} .
$$

It is more convenient in this case to use Equation (64). Integration in Equation (64) over $z$ can be replaced by integration over $d r=a^{\prime}(z) d z$ at the boundaries $z= \pm g / 2$. This gives

$$
\begin{aligned}
Z_{l}^{(0)}(k)= & \left(k Z_{0} / 2 \pi\right) \int d p \int_{a}^{b} d r \int_{a}^{b} d r^{\prime} \sin ^{2}[(g / 2)(k-p)] \\
& \times\left[\theta\left(r-r^{\prime}\right) J_{1}\left(\Omega r^{\prime}\right) H_{1}^{(1)}(\Omega r)+\theta\left(r^{\prime}-r\right) J_{1}(\Omega r) H_{1}^{(1)}\left(\Omega r^{\prime}\right)\right] .
\end{aligned}
$$

Integrals over $r, r^{\prime}$ can be calculated explicitly:

$$
\begin{aligned}
Z_{l}^{(0)}(k)= & \left(k Z_{0} / 2 \pi\right) \int\left(d p / \Omega^{2}\right) \sin ^{2}[(g / 2)(k-p)] \\
& \times\left\{\left[J_{0}(\Omega b)-J_{0}(\Omega a)\right] H_{0}^{(1)}(\Omega b)\right. \\
& \left.-\left[H_{0}^{(1)}(\Omega b)-H_{0}^{(1)}(\Omega a)\right] J_{0}(\Omega a)+(2 i / \pi) \ln (b / a)\right\} .
\end{aligned}
$$


The impedance for a shallow cavity is inductive $Z_{l}^{(0)}=-i k L=-i k £ / c$. The inductance $€$ (in $\mathrm{cm}$ ), calculated from Equation (83), is depicted in Figure 3 as a function of $g /(b-a)$. The integration has been performed for a cavity with radii $b=4.2 \mathrm{~cm}, b-a=0.2 \mathrm{~cm}$. The inductance calculated from Equation (74) is shown by the dashes. The agreement of the results found above for small $g /(b-a)$ also takes place for a wider range of gaps, up to $g \simeq a$.

The real part of the impedance is given by the interval $-k<p<k$ :

$$
\begin{aligned}
\operatorname{Re} Z_{l}^{(0)}(k)= & \left(k Z_{0} / 2 \pi\right) \int_{-k}^{k}\left[d p /\left(k^{2}-p^{2}\right)\right] \sin ^{2} \\
& \times[(g / 2)(k-p)]\left[J_{0}(\Omega a)-J_{0}(\Omega b)\right]^{2} .
\end{aligned}
$$

To estimate the integral Equation (84) in the high-frequency limit $k a \gg 1$ for $b \gg a \simeq g$, we can neglect $J_{0}(\Omega b)$. The main contribution at high frequencies $k a \gg 1, k b \gg 1$ is given by the range of $p$ for which $g(k-p) \geq 1, \Omega a \gg 1$. Using an asymptotic expression for the Bessel function, we obtain ${ }^{11}$ the Dome-Lawson result of Equation (81).

For very large gaps $g$, the impedance depends not on $g$, but on both radii. The impedance can be obtained from Equation (84) in the limit $g \rightarrow \infty$, replacing $\sin ^{2}[\ldots]$ by its average value $1 / 2$. For the pipes of equal radii at large $z$,

$$
\operatorname{Re} Z_{l}^{(0)}(k)=\left(k Z_{0} / 4 \pi\right) \int\left[d p /\left(k^{2}-p^{2}\right)\right]\left[J_{0}(\Omega b)-J_{0}(\Omega b)\right]^{2} .
$$

The main contribution for $k a \gg 1, k b \gg 1, b \gg a$ is given by the interval $(1 / a) \gg \Omega \gg(1 / b)$ :

$$
\operatorname{Re} Z_{l}^{(0)}(k)=\left(Z_{0} / 4 \pi\right) \ln k a .
$$

For small $b, k b<1 ; k a$ should be replaced by $b / a$, giving an impedance similar to the impedance of a step,

$$
\operatorname{Re} Z_{l}^{(0)}(k)=\left(Z_{0} / 4 \pi\right) \ln (b / a)
$$

Transition from the regime of a cavity to the regime of a step occurs ${ }^{14}$ at $g \simeq$ $k(b-a)^{2}$.

The result of a numerical integration of Equation (84) is shown in Figure 4a. The real part of the impedance is a linear function of the Dome-Lawson parameter $(1 / 2 \pi a)(g / \pi k)^{1 / 2}$. Results in the "cavity regime" $g \gg k(b-a)^{2}$ are independendent of $b$. Figure $4 \mathrm{~b}$ shows that there is a smooth transition from the regime of a cavity to the regime of a step. Transition frequency depends on $(b-a) / a$. 


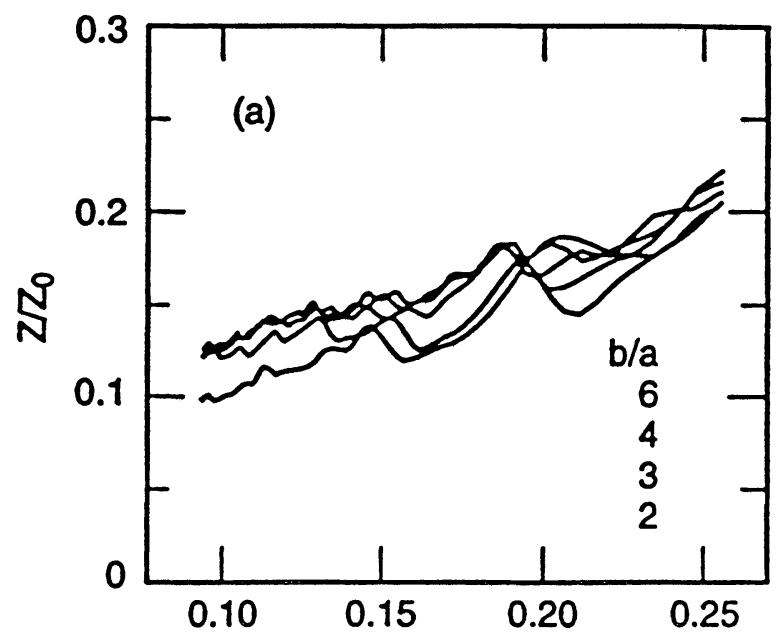

FIGURE 4(a):

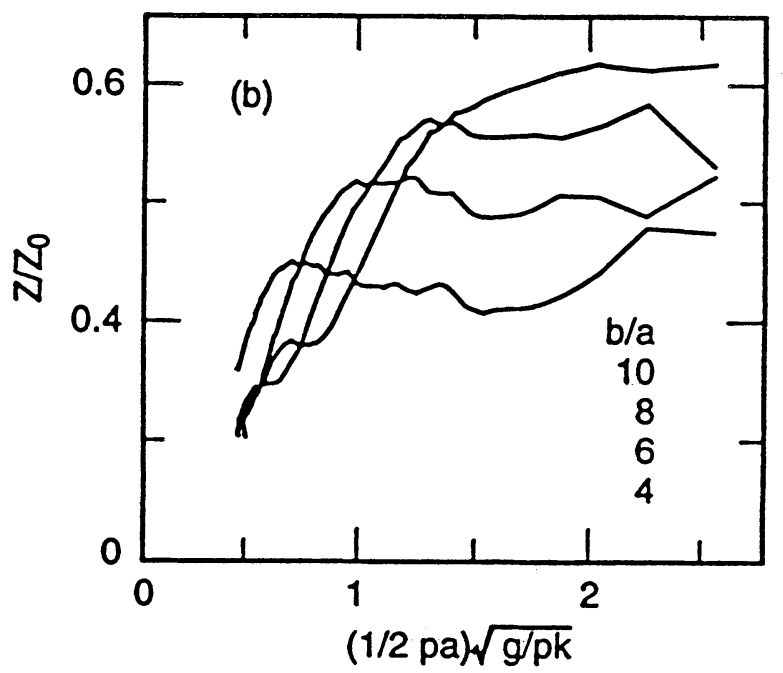

FIGURE 4(b):

FIGURE 4: (a)The real part of the longitudinal impedance of a cavity, Equation (84), as a function of the Dome-Lawson parameter. Results are essentially independent of the $b ; g / a=3.0, b / a$ is in the range 2.0-6.0. (b)The same as (a), but for large gaps. The transition from the regime of a cavity to the regime of a step is shown. 


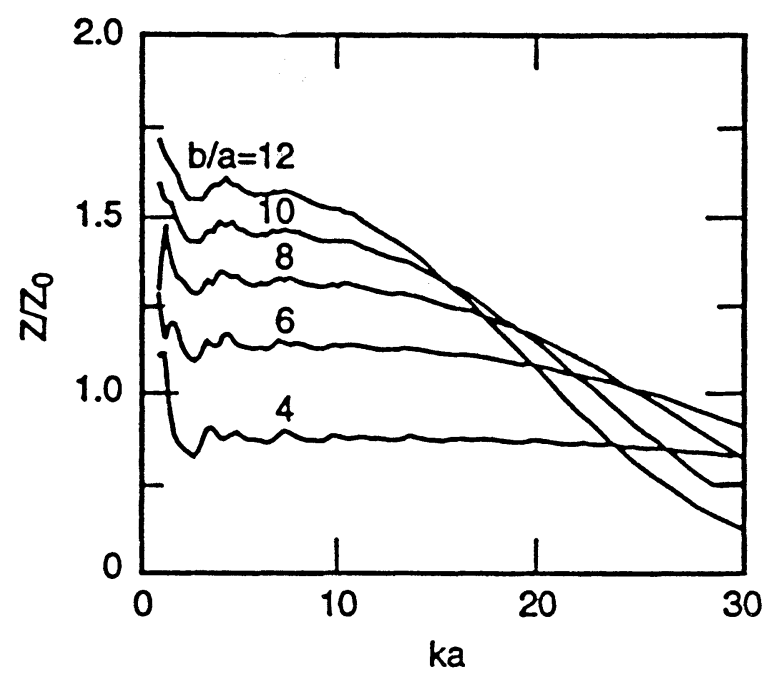

FIGURE 5: The frequency dependence of the impedance of a collimator. The impedance is constant for small $k a$ and rolls off at large frequencies. The roll-off starts at frequencies dependent on the ratio of the radii.

The impedance of a collimator can be derived similarly to the impedance of a cavity, but with the correction discussed in the zero-order approximation,

$$
\begin{aligned}
Z_{l}^{(0)}(k)= & \left(k Z_{0} / 2 \pi\right) \int\left(d p / \Omega^{2}\right) \\
& \times\left\{J_{0}(\Omega a)\left[H_{0}^{(1)}(\Omega a)-H^{(1)}(\Omega b)\right]\right. \\
& \left.-H_{0}^{(1)}(\Omega b)\left[J_{0}(\Omega a)-J_{0}(\Omega b)\right]+(2 i / \pi) \ln (b / a)\right\} .
\end{aligned}
$$

The real part of the impedance is given by the interval $-k<p<k$ :

$$
\operatorname{Re} Z_{l}^{(0)}(k)=\left(k Z_{0} / 2 \pi\right) \int_{-k}^{k}\left[d p /\left(k^{2}-p^{2}\right)\right]\left[J_{0}(\Omega a)-J_{0}(\Omega b)\right]^{2} .
$$

The impedance calculated from this formula is shown in Figure 5.

\subsection{Impedance of a periodic array of cavities}

Consider an array of $M$ identical cells made by irises in a straight pipe. The radius of the pipe is $b$, the radius of the opening in the washers is $a$, the thickness of the iris is $l$, and the length of a period is $d$. 
The real part of the impedance can be obtained from Equation (65):

$$
\operatorname{Re} Z_{l}^{(0)}(k)=\left(k Z_{0} M / 2 \pi\right) \int_{k}^{k}\left[d p /\left(k^{2}-p^{2}\right)\right]\left[J_{0}(\Omega b)-J_{0}(\Omega b)\right]^{2} F(k, p) .
$$

This differs from the result of Equation (84) for a single cell by a form factor $F(k, p)$, describing interference of the waves generated by a bunch in different cells:

$$
F(k, p)=(1 / 4 \pi) \sum_{i, j=1}^{M}(-1)^{i-j} \exp \left\{i(k-p)\left(z_{i}-z_{j}\right)\right\} .
$$

Here $z_{i}$ are the coordinates of the irises:

$$
z_{2 n+1}=n d, n=0,1, \ldots, M-1, \quad z_{2 n}=n d-l, n=1,2, \ldots M .
$$

The sum, Equation (76), can be calculated. Usually $l \ll d$, and

$$
F(k, p)=(1 / \pi M) \sin ^{2}[(g / 2)(k-p)] \sin ^{2}\{[(k-p) d / 2] M\} .
$$

For small $M \simeq 1$, the impedance per cell is the same as that for a single cell. At high frequencies, provided $k a^{2} / d \gg>1$, it is given by Equation (81).

For $M \gg 1$, the impedance per cell from Equation (90) is the convolution of two sharp functions. The first function in the integrand of Equation (90) is the same as for a single cavity and has a sharp maximum for $|k-p| \leq\left(1 / k a^{2}\right)$. The second form factor (92) has a maximum for $|k-p| \leq(1 / M d)$. The frequency behavior of the impedance depends on the parameter $k a^{2} / M d$. For a structure with fixed total length $M d$, there are always very large $k a$ where the first function dominates and impedance per cell is the same as for a single cell. However, there is an intermediate range of frequencies where $M \gg k a^{2} / d$, where the second function is dominant. For such frequencies impedance rolls off as $k^{-3 / 2}$ instead of $k^{-1 / 2}$. More discussion on this can be found in the original paper ${ }^{15}$. The same result has been obtained by R.Gluckstern ${ }^{16}$.

\subsection{Impedance of a taper}

The radius of a taper varies linearly from $a$ to $b>a$ at distance $L$,

$$
r(z)=a, z \leq 0 ; \quad r(z)=a+a^{\prime} z, 0<z<L ; \quad r(z)=a+b L, z>L .
$$

The longitudinal impedance is

$$
Z_{l}^{(0)}(k)=\left(Z_{0} / \pi\right) \ln (b / a)+\left\{\left[k Z_{0}\left(a^{\prime}\right)^{2} / 8 \pi\right\} S(k),\right.
$$


where

$$
S(k)=\left.\int d p \int_{0}^{L} d z d z^{\prime} \exp \left\{i(p-k)\left(z-z^{\prime}\right)\right\}\left[G_{1, p}\left(z, z^{\prime}\right)\right]\right|_{r=a(z), r^{\prime}=a\left(z^{\prime}\right)} .
$$

The cuts in the plane $p$ are defined so that $\Omega=i\left(p^{2}-k^{2}\right)^{1 / 2}$ for $p>k$ and $p<-k$, and the contour of integration is below the cuts. Because there are no singularities in the lower plane of $p$, the integration over $p$ gives zero if $z<z^{\prime}$. Hence,

$$
S(k)=\int d p \int_{0}^{L} d z \int_{0}^{z} d z^{\prime} \exp \left\{i(p-k)\left(z-z^{\prime}\right)\right\} J_{1}\left[\Omega a\left(z^{\prime}\right)\right] H_{1}^{(1)}[\Omega a(z)] .
$$

We will study this expression at high frequencies, which give the main contribution to the energy loss.

First, consider a taper with large angle $\alpha, \tan \alpha=a^{\prime}, a^{\prime} \lesssim 1$. Equation (96) in this case can be simplified using asymptotic expressions for the Bessel functions and retaining the slowest-oscillating exponential function:

$$
S(k)=\int d q \int_{0}^{L} d z \int_{0}^{z} d z^{\prime}\left(2 /\left\{\pi \Omega\left[a(z) a\left(z^{\prime}\right)\right]\right\}^{1 / 2}\right) \exp \left\{-i\left(z-z^{\prime}\right) \psi\left(z, z^{\prime}, q\right)\right\}
$$

where $q=k-p$, and $\psi=q-a^{\prime}[q(2 k-q)]^{1 / 2}$. The integral over $q$ can be calculated by a saddle-point method, expanding $\psi$ around $q_{0}=k a^{\prime 2} / 2$, provided $k a\left|a^{\prime}\right| \gg 1$. That gives

$$
\psi=-\left(k a^{2} / 2\right)+\left[\left(q-q_{0}\right)^{2} / 4 q_{0}\right], \quad \Omega \simeq\left|k a^{\prime}\right|
$$

and

$$
S(k)=2(2 / i \pi k) \int_{0}^{L}[d z / a(z)] \int_{0}^{z}\left[d z^{\prime} /\left(z-z^{\prime}\right)^{1 / 2}\right] \exp \left\{i\left(k a^{\prime 2} / 2\right)\left(z-z^{\prime}\right)\right\} .
$$

Significant values of $\left|z-z^{\prime}\right| \sim 1 /\left(k a^{\prime 2}\right)$ are small provided $k a^{\prime 2} L \gg 1$. This reduces the integral to

$$
S(k)=\left(8 /\left|k a^{\prime}\right|\right) \int_{0}^{L}[d z / a(z)]=\left(8 / k a^{\prime 2}\right) \ln (b / a) .
$$

The impedance obtained from Equations (94) and (100) is the same as the impedance of a step with radii $a, b$ :

$$
Z_{l}^{(0)}(k)=\left(Z_{0} / 2 \pi\right) \ln (b / a)
$$


The result is valid if

$$
k a\left|a^{\prime}\right| \gg 1 \quad \text { and } \quad k\left|a^{\prime}(b-a)\right| \gg 1 .
$$

If $k a\left|a^{\prime}\right| \gg 1$, but $b-a$ are so small that $k\left|a^{\prime}(b-a)\right| \ll 1$, the integral of Equation (99) gives

$$
\begin{aligned}
S(k) & =2(2 / i \pi k)^{1 / 2} \int_{0}^{L} d z /\left(a+a^{\prime} z\right) \int_{0}^{z}\left[d z^{\prime} /\left(z-z^{\prime}\right)^{1 / 2}\right] \\
& =(8 / 3 \pi a)(2 \pi / i k)^{1 / 2}\left[(b-a) / a^{\prime}\right]^{3 / 2} .
\end{aligned}
$$

From this consideration, it follows that it is sufficient to study a shallow taper with $\left|a^{\prime}\right| \ll 1$.

In this case it is more convenient to write the impedance in the form of Equation $(65)$ and write $S(k)$ in the form

$$
S(k)=\int\left(d \phi / i \pi a^{2}\right) \int_{a}^{b} d r d r^{\prime} \exp \left\{-i k\left(z-z^{\prime}\right)\right\}\left[\exp \left\{i k\left|\vec{r}-\vec{r}^{\prime}\right|\right\} /\left|\vec{r}-\vec{r}^{\prime}\right|\right] \cos (\phi)
$$

The difference

$$
\left|\vec{r}-\vec{r}^{\prime}\right|=\left(1 /\left|a^{\prime}\right|\right)\left[\left(r-r^{\prime}\right)^{2}\left(1+a^{2}\right)+2 r r^{\prime} a^{\prime 2}(1-\cos \phi)\right]^{1 / 2}
$$

can be expanded for small $\left|a^{\prime}\right| \ll 1$,

$$
\begin{aligned}
\left|\vec{r}-\vec{r}^{\prime}\right|= & {\left[\left(1+a^{2}\right) /\left|a^{\prime}\right|\right]^{1 / 2}\left|r-r^{\prime}\right| } \\
& +\left\{\left[r r^{\prime}\left|a^{\prime}\right|(1-\cos \phi)\right] /\left[\left|r-r^{\prime}\right|\left(1+a^{\prime 2}\right)^{1 / 2}\right]\right\},
\end{aligned}
$$

provided

$$
\left(r-r^{\prime}\right)^{2} \gg r^{2} a^{\prime 2}
$$

Then

$$
S(k)=(1 / i \pi) \int_{a}^{b}\left(d r d r^{\prime} /\left|r-r^{\prime}\right|\right) \int\left\{d \phi /\left[\left|a^{\prime}\right|\left(1+a^{2}\right)^{1 / 2}\right]\right\} \exp \{-i \phi+i \Psi\},
$$

where

$$
\begin{aligned}
\Psi= & -\left(k / a^{\prime}\right)\left(r-r^{\prime}\right)+\left\{\left[k\left(1+a^{\prime 2}\right)^{1 / 2}\right] /\left|a^{\prime}\right|\right\}\left|r-r^{\prime}\right| \\
& +\left\{\left[k r r^{\prime}\left|a^{\prime}\right|(1-\cos \phi)\right] /\left[\left|r-r^{\prime}\right|\left(1+a^{2}\right)^{1 / 2}\right]\right\} .
\end{aligned}
$$


The integral over $\phi$ gives the Bessel function. For $\left|a^{\prime}\right| \ll 1$, the phase $\Psi$ is large and the exponent oscillates rapidly if $r-r^{\prime}<0$ for $a^{\prime}>0$, and $r-r^{\prime}<0$ for $a^{\prime}<0$. Consider, for example, $a^{\prime}>0$. Then $r>r^{\prime}$ are significant, for which

$$
\Psi=\left(k\left|a^{\prime}\right| / 2\right)\left(r-r^{\prime}\right)+\left\{\left[k r r^{\prime}\left|a^{\prime}\right|(1-\cos \phi)\right] /\left[\left|r-r^{\prime}\right|\left(1+a^{2}\right)^{1 / 2}\right]\right\} .
$$

The first term here is negligibly small provided

$$
\lambda \equiv k a\left|a^{\prime}\right| \ll 1 .
$$

With the new variables $y=r / a$ and $x=\left(r-r^{\prime}\right) / a$, Equation (106) takes the form

$$
S(k)=\left(a / i \pi\left|a^{\prime}\right|\right) \int(d x d y /|x|) J_{1}\left(\lambda y^{2} /|x|\right) \exp \left\{i \lambda\left(y^{2} /|x|\right)\right\} .
$$

Here the limits of the integration are

$$
1<y<p \equiv(b / a), \quad y-p<x<y-1 .
$$

One of the integrals can be calculated, introducing the new variables $\tau=y^{2} / x, \xi=y$, and changing the order of integration. The limits of integration over $\tau, \xi$ are, for $p<2$,

$$
\left[p^{2} /(p-1)\right]<\tau<\infty, \quad(\tau / 2)-\left[\left(\tau^{2} / 4\right)-\tau\right]^{1 / 2}<\xi<p,
$$

and for $p>2$ there are two integrals with the limits

$$
4<\tau<\left[p^{2} /(p-1)\right], \quad(\tau / 2)-\left[\left(\tau^{2} / 4\right)-\tau\right]^{1 / 2}<\xi<(\tau / 2)+\left[\left(\tau^{2} / 4\right)-\tau\right]^{1 / 2},
$$

and

$$
\left[p^{2} /(p-1)\right]<\tau<\infty, \quad(\tau / 2)-\left[\left(\tau^{2} / 4\right)-\tau\right]^{1 / 2}<\xi<p .
$$

Then the function $S(k)$ is given by the integral

$$
\begin{aligned}
S(k)= & \left(a / i \pi\left|a^{\prime}\right|\right)\left(\theta(p-2) \int_{4}^{Q(p)}(d \tau / \tau)\left[\left(\tau^{2} / 4\right)-\tau\right]^{1 / 2} J_{1}(\lambda \tau) \exp \{i \lambda \tau\}\right. \\
& \left.+\int_{Q(p)}^{\infty}(d \tau / \tau)\left\{p-(\tau / 2)+\left[\left(\tau^{2} / 4\right)-\tau\right]^{1 / 2}\right\} J_{1}(\lambda \tau) \exp \{i \lambda \tau\}\right)
\end{aligned}
$$

where $p=b / a$ and $Q(p)=\left[p^{2} /(p-1)\right]$.

The integral (109) can further be estimated in two extreme cases. If $\lambda Q(p) \ll 1$, impedance (94),(109) increases linearly with $\left|a^{\prime}\right|$ :

$$
Z_{l}^{(0)}(k)=-i\left(Z_{0} \lambda / 8 \pi\right) \int_{0}^{\infty}(d x / x) J_{1}(x) \exp \{i x\}+o\left(\lambda^{2}\right) .
$$



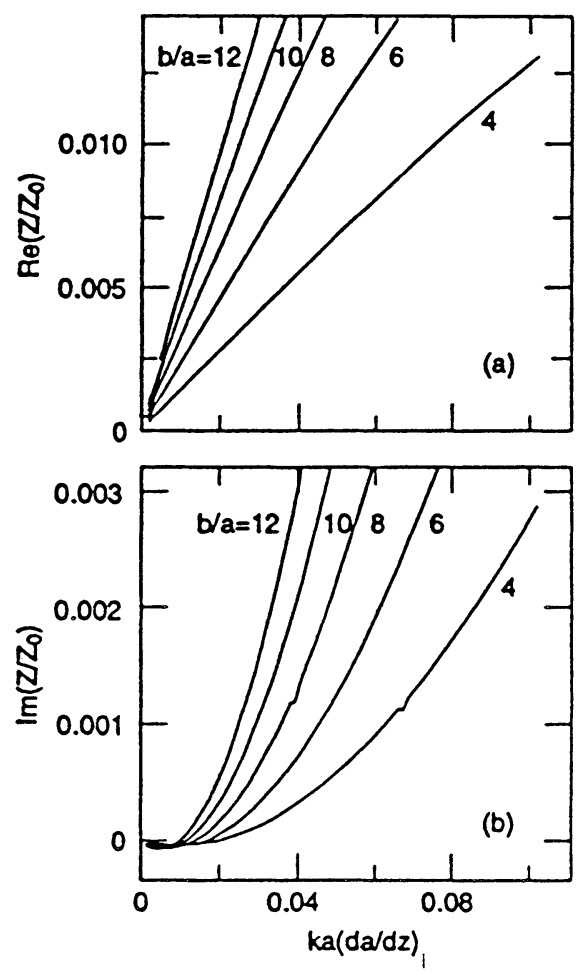

FIGURE 6: The real (a) and imaginary (b) parts of the longitudinal impedance of a taper, with large $p=b / a$.

This result is confirmed by the numeric integration of Equation (109); see Figure 6a,b.

For very small $p-1=(b-a) / a \ll 1$, there is another regime where

$$
\lambda \ll 1, \quad \text { but } \quad\left[\lambda p^{2} /(p-1)\right] \gg 1 .
$$

The impedance in this case is

$$
\begin{aligned}
Z_{l}^{(0)}(k)= & {\left[Z_{0}(1+i) /(4 \pi)^{2}\right](\lambda / \pi)^{1 / 2} } \\
& \times \int_{Q(p)}^{\infty}\left(d x / x^{3 / 2}\right)\left\{p-(x / 2)+\left[\left(x^{2} / 4\right)-x\right]^{1 / 2}\right\},
\end{aligned}
$$

and is proportional to the $\left(a^{\prime}\right)^{1 / 2}$ term. This behavior has previously been found numerically. ${ }^{17}$ 

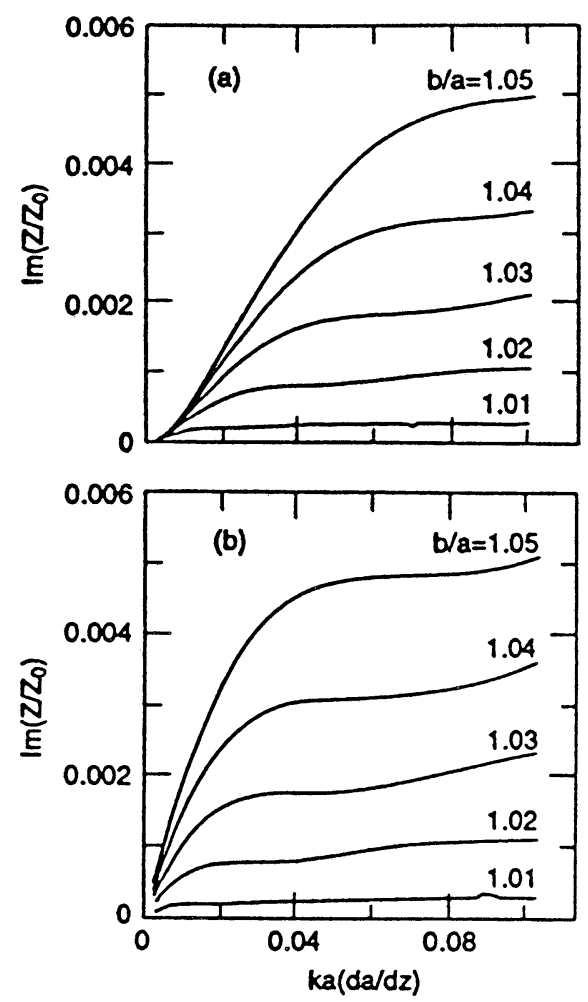

FIGURE 7: The real (a) and imaginary (b) parts of the longitudinal impedance of a taper, for small $(p-1) \ll 1$.

Equation (111) is compared with the numerical integration of Equation (109) for small $(p-1) \ll 1$; see Figure 7a,b. The transition from small $p-1$ to $p-1 \simeq 1$ is depicted in Figure 8.

\section{EXAMPLES OF TRANSVERSE IMPEDANCE}

The transverse impedance is given by Equations (5) and (71):

$$
\begin{aligned}
Z_{\perp}(k)= & \left(Z_{0} / 8 \pi\right) \int d p \int d z d z^{\prime} \exp \left\{i(k-p)\left(z^{\prime}-z\right)\right\} \\
& \times\left[a^{\prime}(z) a^{\prime}\left(z^{\prime}\right) / a(z) a\left(z^{\prime}\right)\right]\left[G_{2, p}\right]_{r=a(z), r^{\prime}=a\left(z^{\prime}\right)} .
\end{aligned}
$$




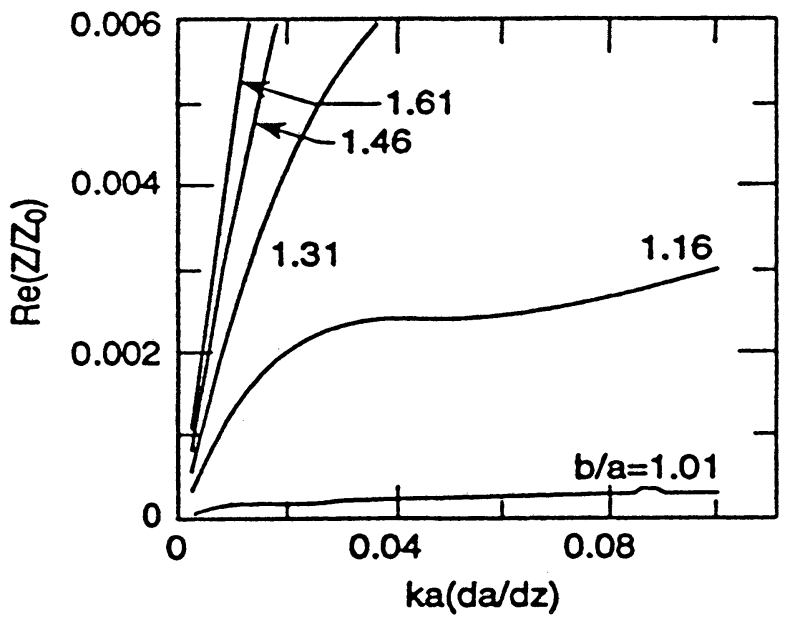

FIGURE 8: The same as in Figure 6(a), for the transition from $(p-1) \ll 1$ to $(p-1) \simeq 1$.

or

$$
\begin{aligned}
Z_{\perp}(k)= & -i\left(Z_{0} / 2 \pi\right) \int d \phi^{\prime} \cos 2\left(\phi-\phi^{\prime}\right) \int d z d z^{\prime} \exp \left\{-i k\left(z-z^{\prime}\right)\right\} \\
& \times\left[a^{\prime}(z) / a(z)\left[a^{\prime}\left(z^{\prime}\right) / a\left(z^{\prime}\right)\right]\left[G_{k}\left(\vec{R}, \vec{R}^{\prime}\right)\right]_{r=a(z), r^{\prime}=a\left(z^{\prime}\right)} .\right.
\end{aligned}
$$

We consider here several examples.

\subsection{Impedance of a cavity with attached tubes}

We consider first Equation (71), for a cavity with attached tubes:

$$
r(z)=a,|z|>g / 2, \quad r(z)=b,|z|<g / 2, \quad b>a .
$$

For a shallow cavity of low frequency $k(b-a) \ll 1$, the impedance is proportional to to the gap length $g$,

$$
Z_{\perp}(k)=-\frac{2 i g(b-a)}{c a^{3}},
$$

if $g \ll 2(b-a) 4$. For large gaps $g \gg 2(b-a)$, dependence on the gap length is logarithmic,

$$
Z_{\perp}(k)=-\frac{2 i}{\pi} \frac{(b-a)^{2}}{c a^{3}}\left\{\ln \frac{g}{2(b-a)}+1\right\}, \quad \frac{k g}{2}<1,
$$

and is independent of $g$ for $k g / 2>1$. 
In the high-frequency limit $k g \gg 1, k a \gg 1$, it is more convenient to use Equation (72), where the integrals over $d r=a^{\prime}(z) d z$ can be calculated using the expressions

$$
\left\{\left[J_{2}(\Omega r)\right] / r\right\}=-(1 / \Omega)(\partial / \partial r)\left\{\left[J_{1}(\Omega r)\right] / r\right\},
$$

and

$$
H_{2}^{(1)}(z) J_{1}(z)-J_{2}(z) H_{1}^{(1)}(z)=-(2 i / \pi z)
$$

That gives

$$
\begin{aligned}
Z_{\perp}(k)= & \left(Z_{0} / 2 \pi\right) \int\left(d p / \Omega^{2}\right) \sin ^{2}[(g / 2)(k-p)]\left\{(i / \pi)\left[\left(1 / b^{2}\right)-\left(1 / a^{2}\right)\right]\right. \\
& -\left\{\left[H_{1}^{(1)}(\Omega b)\right] / b\right\}\left(\left\{\left[J_{1}(\Omega b)\right] / b\right\}-\left\{\left[J_{1}(\Omega a)\right] / a\right\}\right) \\
& +\left\{\left[J_{1}(\Omega a)\right] / a\right\}\left(\left\{\left[H_{1}^{(1)}(\Omega b)\right] / b\right\}-\left\{\left[H_{1}^{(1)}(\Omega a)\right] / a\right\}\right) .
\end{aligned}
$$

The real part of the impedance again is given by the interval $-k<p<k$ :

$$
\begin{aligned}
\operatorname{Re} Z_{\perp}(k)= & \left(Z_{0} / 2 \pi k\right) \int_{-1}^{1}\left[d x /\left(1-x^{2}\right)\right] \sin ^{2}[(k g / 2)(1-x)] \\
& \times\left(\left\{J_{1}\left[k b\left(1-x^{2}\right)^{1 / 2}\right] / b\right\}-\left\{J_{1}\left[k a\left(1-x^{2}\right)^{1 / 2}\right] / a\right\}\right)^{2} .
\end{aligned}
$$

The behavior of the impedance given by Equation (116) depends on the ratio $g / a$. For $g<k a^{2}, k g \gg 1$ this impedance increases as $k^{3}$ for $k a \ll 1$, reaches maximum at $k a \simeq 1$, and rolls-off as $(1 / k a)^{3 / 2}$ for large $k a$. For $g>k a^{2}$, this impedance increases as $k^{3}$ for $k a \ll 1$, reaches maximum at $k a \simeq 1$, and rolls off as $(1 / k a)$ for large $k a$.

The results of numerical integration of Equation (116) are shown in Figure 9a,b.

\subsection{Transverse impedance of a taper}

For a taper, the impedance is given by Equations (5) and (72):

$$
\begin{aligned}
Z_{\perp}(k)= & -\left[i Z_{0} / 2(2 \pi)^{2}\right] \int d \phi^{\prime} \cos \left(\phi-\phi^{\prime}\right) \int_{a}^{b}(d r / r)\left(d r^{\prime} / r^{\prime}\right) \\
& \times \exp \left\{-i k\left(z-z^{\prime}\right)\right\}\left[\left(\exp \left\{i k\left|\vec{r}-\vec{r}^{\prime}\right|\right\}\right) /\left(\left|\vec{r}-\vec{r}^{\prime}\right|\right)\right]
\end{aligned}
$$

where $z-z^{\prime}=\left(r-r^{\prime}\right) / a^{\prime}$. 

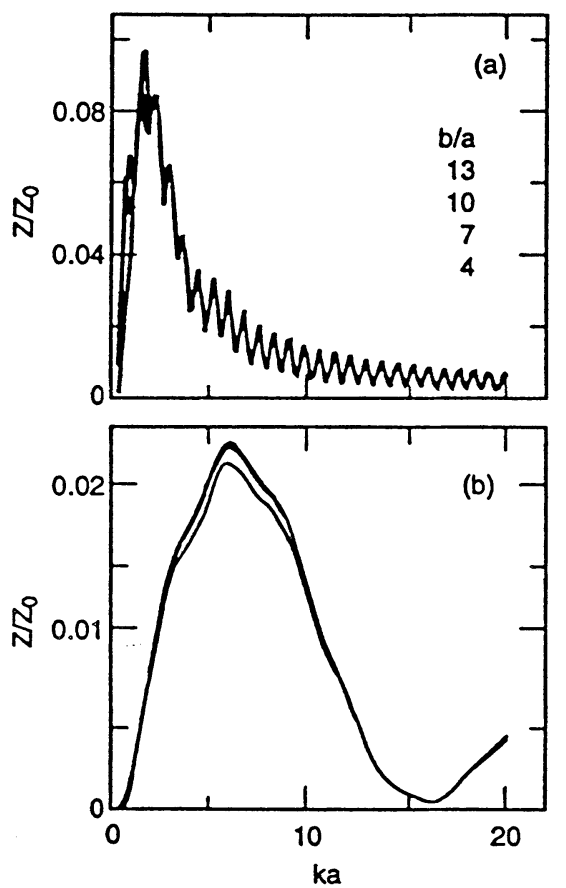

FIGURE 9: The real part of the transverse impedance of a cavity, (a) for $g / a=4.0$, and (b) for small $g / a=0.2$. These results are independent of $b / a$, which is in the range 4.0-13.0 for both (a) and (b). The curve for (b) is broader and rolls off slower than for (a).

Expanding $\left|\vec{r}-\vec{r}^{\prime}\right|$ as in Equation (105), we can calculate the integral over the angle $\phi$. This gives the Bessel function $J_{2}$. For a shallow taper $\left|a^{\prime}\right| \ll 1$, the main contribution is given by

$$
r\left|a^{\prime}\right| \ll\left|r^{\prime}-r\right| \ll r \simeq r^{\prime} .
$$

With a proper choice of variables, it is possible to carry out one more integration in the same way as for the longitudinal impedance of a taper. The impedance is given by the remaining integral:

$$
\begin{aligned}
Z_{\perp}(k)= & -\left(i Z_{0}\left|a^{\prime}\right| / 4 \pi a\right)\{\theta(p-2) \\
& \times \int_{4}^{Q(p)}(d \tau / \tau) J_{2}(\lambda \tau) \exp \{i \lambda \tau\}\left[\left(1 / \xi_{-}\right)-\left(1 / \xi_{+}\right)\right] \\
& \left.+\int_{Q(p)}^{\infty}(d \tau / \tau) J_{2}(\lambda \tau) \exp \{i \lambda \tau\}\left[\left(1 / \xi_{-}\right)-(1 / p)\right]\right\}
\end{aligned}
$$


where $p=b / a, Q(p)=\left[p^{2} /(p-1)\right]$, and

$$
\xi_{ \pm}=(\tau / 2) \pm\left[\left(\tau^{2} / 4\right)-\tau\right]^{1 / 2} .
$$

The result, Equation (118), is valid if

$$
\left|a^{\prime}\right| \ll \lambda \equiv k a\left|a^{\prime}\right| \ll 1 .
$$

\section{CONCLUSION}

The perturbation method described above allows derivation of the general expressions for the longitudinal [Equations (65), (72)] and transverse [Equation (113)] impedances for axially symmetric structures with arbitrary variation of the radius along the structure.

It is shown that the formulas reproduce, in a systematic way, numerous previously known results and obtain new results.

The longitudinal impedance is found for these geometries:

\begin{tabular}{l} 
Parameter \\
\hline (a) Hole \\
(b) Slot \\
(c) Step \\
(d) Shallow cavity \\
(e) Shallow Collimator \\
(f) Cavity with pipes \\
(g) Taper
\end{tabular}
Equation

(99), (95) and Figs 6-8

Transverse impedance is considered for these geometries.

\begin{tabular}{l} 
Parameter \\
\hline (a) Hole \\
(b) Step \\
(c) Shallow cavity \\
(d) Taper
\end{tabular}

\begin{tabular}{c} 
Equation \\
\hline$(44)$ \\
$(57)$ \\
$(116)$ \\
$(118)$ \\
\hline
\end{tabular}

This method allows us to obtain all these results in a unified way as extreme cases of the same formula, and to demonstrate the transition from one case to another; for example, from the regime of a cavity to the regime of a step, Figure 4, or from a single cavity to a periodic array, Equation (92). It seems that the method always works where 
the narrowband impedance is not dominant - in other words, at high frequencies and also for low frequencies, but only for shallow discontinuities. Several new results are obtained, including the longitudinal and transverse impedances of a taper. The method can be generalized to more complicated geometries: for example, for a hole in a beampipe with finite wall thickness or for a structure without axial symmetry.

\section{ACKNOWLEDGEMENTS}

I am thankful for discussions with K. Bane, S. Kheifets, M. Sands, M. Zolotarev, and B. Zotter.

\section{REFERENCES}

1. K. F. L. Bane, P. B. Wilson, and T. Weiland, "Wakefields and wakefield acceleration," SLAC-PUB3528 (1984); S. Heifets and S. Kheifets, "Coupling impedance in modern accelerators," Rev. of Modern Physics (1991) and SLAC-PUB-5297 (1990).

2. T. Weiland, "Comment on wakefield computation in time domain," Nucl. Instrum. Meth. 216, 31-34 (1983).

3. J. D. Jackson, Classical Electrodynamics (Wiley, New York, 1975).

4. A. V. Novokhatsky, preprint, INP, Novosibirsk (1989).

5. S. Heifets, "Diffraction model of the high-frequency impedance," Phys. Rev. D 40, 9, 3097-3106 (1989).

6. H. A. Bethe, "Theory of diffraction by small holes," Phys. Rev. 66 (7-8), 163-182 (1944).

7. S. S. Kurennoy, "On the coupling impedance of a hole or slot," CERN SL/91-29 (AP)/rev (1991).

8. M. Sands, "Energy loss from small holes in the vacuum chamber," PEP-253 (1977).

9. K. L. F. Bane, "The calculated longitudinal impedance of the SLC damping ring," SLAC-PUB-4618 (1988).

10. G. Dome, "Wake potentials of a relativistic point charge," IEEE Trans. Nucl. Sci. NS-32 (5), 25312534 (1985).

11. S. Heifets and S. Kheifets, "High frequency limit of the longitudinal impedance," Part. Accel. 25, (2-4), 61-72 (1990).

12. V. E. Balakin and A. V. Novokhatsky, "VLEPP: longitudinal beam dynamics," in Proceedings of the 12th International Conference on High Energy Accelerators, (Fermilab, 1983), pp. 117-118.

13. S. Kheifets and S. Heifets, "Radiation of a charge in a perfectly conducting cylindrical pipe with a jump in its cross section," in Proceedings of the 1986 Linear Accelerator Conference, SLAC-PUB3695; K. C. D. Chan and R. Schweinfurth, "Beam energy spread induced by beampipe steps," ibid., pp. AT-6:ATN-87-24 (1987).

14. J. J. Bisognano, S. Heifets and B. C. Yunn, "The loss parameters for very short bunches," CEBAFPR-88-005 (1988); P. B. Wilson, LEP-70/62 (1978).

15. S. Heifets and S. Kheifets, "High frequency limit of the longitudinal impedance of an array of cavities," Phys. Rev. D39 (3), 960 (1989).

16. R. L. Gluckstern, "Longitudinal impedance of a periodic structure at high-frequency," Phys. Rev. D39 (9), 2780-2783 (1989).

17. K. Bane, private communication.

18. L. Palumbo, "Analytic calculations of the impedance of a discontinuity," Part. Accel. 25, 201-216 (1990). 